\title{
Axonally derived matrilin-2 induces proinflammatory responses that exacerbate autoimmune neuroinflammation
}

\author{
Anna Jonas, ${ }^{1}$ Stefan Thiem, ${ }^{2}$ Tanja Kuhlmann, ${ }^{3}$ Raimund Wagener, ${ }^{4}$ Attila Aszodi, ${ }^{5}$ Cameron Nowell, ${ }^{2}$ Karin Hagemeier, ${ }^{3}$ \\ Louise Laverick, ${ }^{6}$ Victoria Perreau, ${ }^{1,7}$ Vilija Jokubaitis, ${ }^{1,8}$ Ben Emery, ${ }^{1,7}$ Trevor Kilpatrick, ${ }^{1,7}$ Helmut Butzkueven, ${ }^{1,6,8}$ \\ and Melissa Gresle ${ }^{6,8}$ \\ 'The Florey Institute of Neuroscience and Mental Health, Parkville, Victoria, Australia. ${ }^{2}$ Ludwig Institute for Cancer Research, Melbourne-Parkville Branch, Parkville, Victoria, Australia. \\ ${ }^{3}$ Institute of Neuropathology, University Hospital Muenster, Muenster, Germany. ${ }^{4}$ Center for Biochemistry and Center for Molecular Medicine, Medical Faculty, University of Cologne, Cologne, Germany. \\ ${ }^{5}$ Department of Surgery, Ludwig-Maximilians-University Munich, Munich, Germany. ${ }^{6}$ Department of Medicine, ${ }^{7}$ Department of Anatomy and Neuroscience, and ${ }^{8}$ Melbourne Brain Centre \\ Royal Melbourne Hospital, University of Melbourne, Parkville, Victoria, Australia.
}

\begin{abstract}
In patients with multiple sclerosis (MS) and mice with experimental autoimmune encephalomyelitis (EAE), inflammatory axonal injury is a major determinant of disability; however, the drivers of this injury are incompletely understood. Here, we used the EAE model and determined that the extracellular matrix protein matrilin-2 (MATN2) is an endogenous neuronal molecule that is regulated in association with inflammatory axonal injury. Compared with WT mice, mice harboring a deletion of Matn2 exhibited reduced disease severity and axon damage following induction of EAE. Evaluation of neuronmacrophage cocultures revealed that exogenous MATN2 specifically signals through TLR4 and directly induces expression of proinflammatory genes in macrophages, promoting axonal damage. Moreover, the MATN2-induced proinflammatory response was attenuated greatly in macrophages from Myd88 $\mathrm{KO}$ mice. Examination of brain sections from patients with MS revealed that MATN2 is expressed in lesions but not in normal-appearing white matter. Together, our results indicate that MATN2 is a deleterious endogenous neuroaxonal injury response signal that activates innate immune cells and could contribute to early axonal damage in CNS inflammatory diseases like MS.
\end{abstract}

\section{Introduction}

Axonal injury is an important pathological feature of acute and chronic multiple sclerosis (MS) lesions. It is now widely accepted that immune-mediated axonal damage and loss are major determinants of irreversible neurological disability in patients with MS (1-4). Therefore, there is a need for novel neuroprotective therapies that can be administered as adjuncts to existing immunomodulatory therapies for the treatment or prevention of progressive disease.

Previous studies have shown that following axonal injury, specific neuronal gene transcription and translation events are initiated, with the potential to either reduce or enhance neuronal survival. For example, in MS autopsy tissue, Dutta et al. (5) used RNA microarrays to demonstrate that cortical neurons induce genes encoding components of the neuroprotective ciliary neurotrophic factor signaling pathway, presumably in response to inflammatory demyelination or distant axonal injury. One limitation of human CNS gene expression profiling in MS is that it is by necessity usually performed on autopsy tissue with highly

Authorship note: Helmut Butzkueven and Melissa Gresle contributed equally to this work.

Conflict of interest: The authors have declared that no conflict of interest exists. Submitted: July 10, 2013; Accepted: September 11, 2014

Reference information: / Clin Invest. 2014;124(11):5042-5056. doi:10.1172/JCI71385. heterogeneous disease course and little ongoing inflammation and with variable RNA degradation post-mortem. Therefore, we investigated neuronal gene expression changes during acute inflammatory CNS axon injury using murine myelin oligodendrocyte glycoprotein 35-55-induced ( $\mathrm{MOG}_{35-55}$-induced) experimental autoimmune encephalomyelitis (EAE) (6-8). The analysis of gene expression in the EAE mouse noninflamed motor cortex, a region containing pyramidal neurons with direct axonal projection to the spinal cord, revealed a significantly altered expression of several mRNAs encoding proteins associated with the extracellular matrix (ECM).

Recent studies have shown that various ECM molecules, including biglycan $(9,10)$, versican (11), and hyaluronan (12), function as damage-associated molecular pattern molecules (DAMPs) (13). DAMPs comprise endogenous molecules that are released following tissue injury and signal the innate immune system to initiate and maintain inflammatory responses (14-16). DAMPs are normally sequestered intracellularly or are bound tightly in the ECM and can only become active signaling molecules following release after cell damage or ECM degradation.

In this study, we identified the noncollagenous ECM protein matrilin-2 (MATN2) as an endogenous molecule that is expressed and upregulated by neurons in response to immune-mediated axonal injury. MATN2 is one of four members of the matrilin family, each of which is thought to act as an oligomeric extracel- 
A
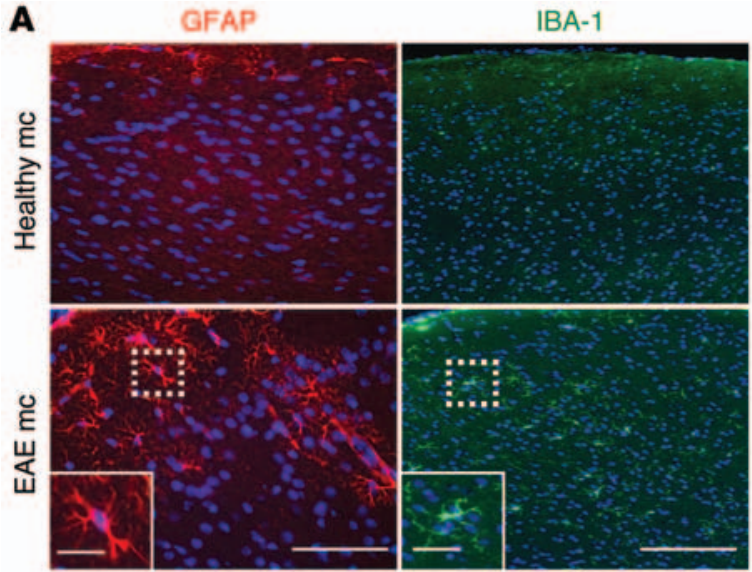

C

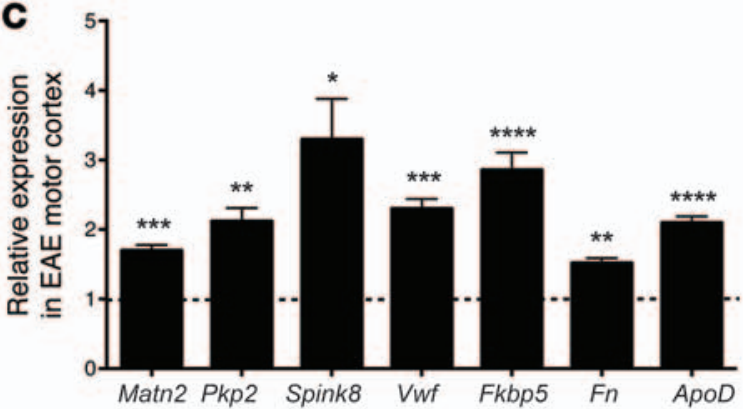

B

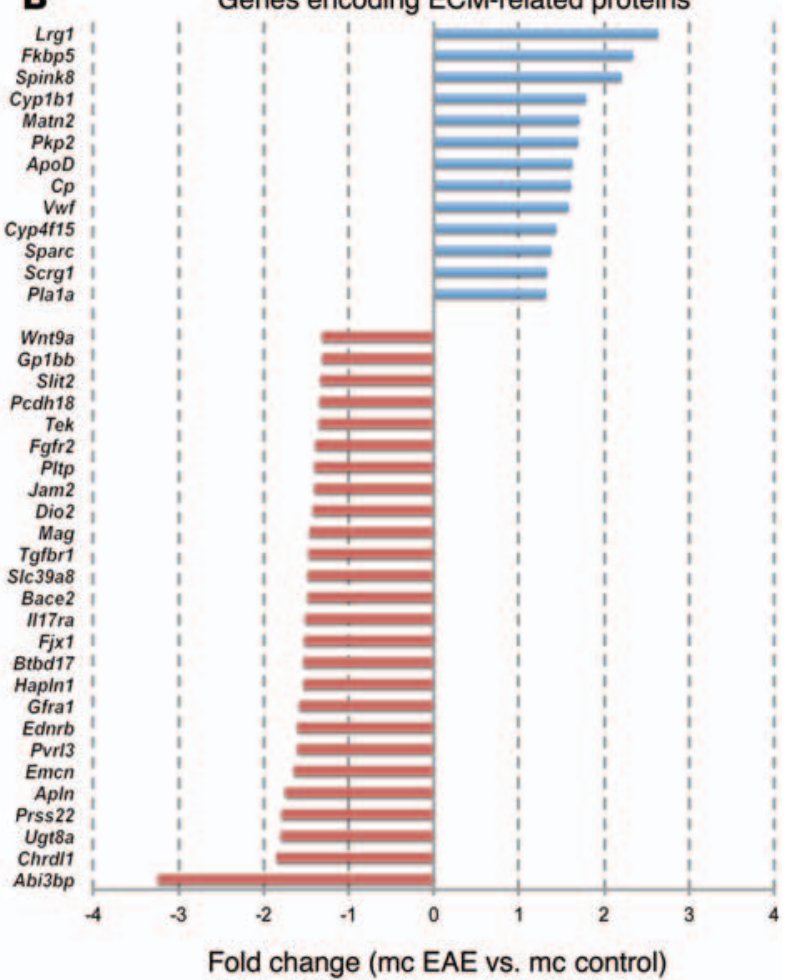

Figure 1. Axonal injury in the spinal cords of EAE mice induces gene expression changes in the motor cortices. (A) Expression of GFAP and IBA-1 in the motor cortices $(\mathrm{mc})$ of healthy and EAE mice is consistent with induction of a glial response in EAE cortices. Nuclei were visualized with DAPI (blue). Scale bar: $100 \mu \mathrm{m}$ (left); $25 \mu \mathrm{m}$ (left inset); $200 \mu \mathrm{m}$ (right); $50 \mu \mathrm{m}$ (right inset). (B) Fold changes $(P<0.05)$ of genes encoding ECM-related proteins differentially expressed in the motor cortices of EAE mice compared with healthy controls identified with microarray analysis. (C) Gene expression changes (mean \pm SEM, qRT-PCR) for indicated transcripts in the motor cortices of EAE mice ( $n=4-8$ per group) relative to healthy control mice (dotted line). ${ }^{*}<<0.05$;

${ }^{* *} P<0.01 ;{ }^{* *} P<0.001 ;{ }^{* * *} P<0.0001$, Student's $t$ test.

lular adaptor protein (17). Outside the CNS, MATN2 is a structural component of the ECM in many tissues, including the skin (18-20). Changes in MATN2 gene expression have further been linked to various inflammation-associated diseases, such as cancer and fibrosis; however, its specific functions remain unclear (21). In the context of peripheral nerve crush injury it has, however, been shown that Matn2 KO mice have delayed and incomplete recovery of neurological function and impaired axonal outgrowth, suggesting a potential role for MATN2 in peripheral nervous system repair (22).

Here, we show a disparate effect of endogenous MATN2 induction in inflammatory CNS injury. We demonstrate that MATN2 is upregulated and released by CNS neurons following acute axonal damage and that extracellular MATN2 induces a proinflammatory response in macrophages/microglia via TLR-associated signaling pathways, thus exacerbating tissue injury.

\section{Results}

Axonal injury in the spinal cords of EAE mice alters gene expression in the motor cortex. Although numerous microarray experiments have been conducted to identify differentially regulated genes in EAE (23), most of these studies have focused on the local lesion environment in the spinal cord. In order to identify genes potentially regulated in neurons in response to more distal neuroinflammation or axonal damage, we studied gene expression in the motor cortices of C57BL/6 mice induced with $\mathrm{MOG}_{35-55}$ EAE. Although there is little lesion activity in the cortex in this model (24), the large pyramidal motor neurons of the motor cortex (corticospinal motoneurons) project their axons through the severely infiltrated spinal cord. To first verify our hypothesis that inflammatory axonal injury within the spinal cord produces a response in the motor cortex, we performed immunohistochemistry (IHC) on coronal sections collected from severely diseased EAE mice (grade 3, day 21 after induction) and healthy mice. We observed increased staining of activated astrocytes (GFAP) and microglia (IBA-1) (Figure 1A), consistent with induction of a glial response in the EAE cortex, but CD3-positive T cells or inflammatory lesions were absent (data not shown). We then compared gene expression in motor cortices of healthy and severely affected EAE mice (grade 3-3.5, EAE day 21) using microarray technology. We identified 132 differentially regulated genes $(P<0.0005$, fold change $\geq 1.3), 52$ with increased and 80 with decreased expression in EAE compared with that in healthy control cortex (Supplemental Table 1; supplemental material available online with this article; doi:10.1172/JCI71385DS1), with a maximum relative expression change of 3.9 -fold. Clustering according to predicted location of gene products showed that 39 of the 132 genes $(29.5 \%)$ were associated with the ECM (Figure 1B). We therefore decided to further assess the most highly regulated ECM-associated genes and validated their 
A

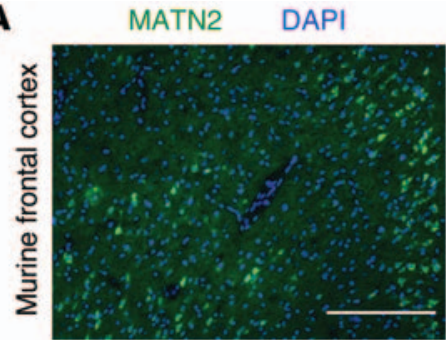

C

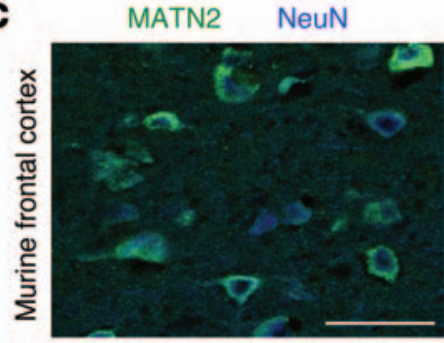

D

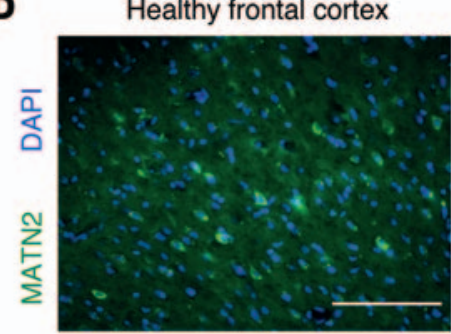

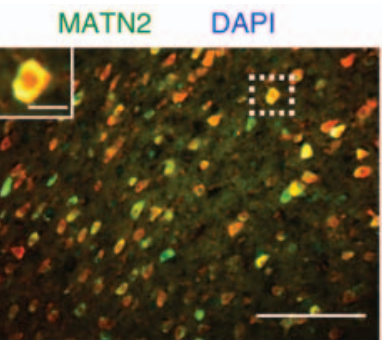

B
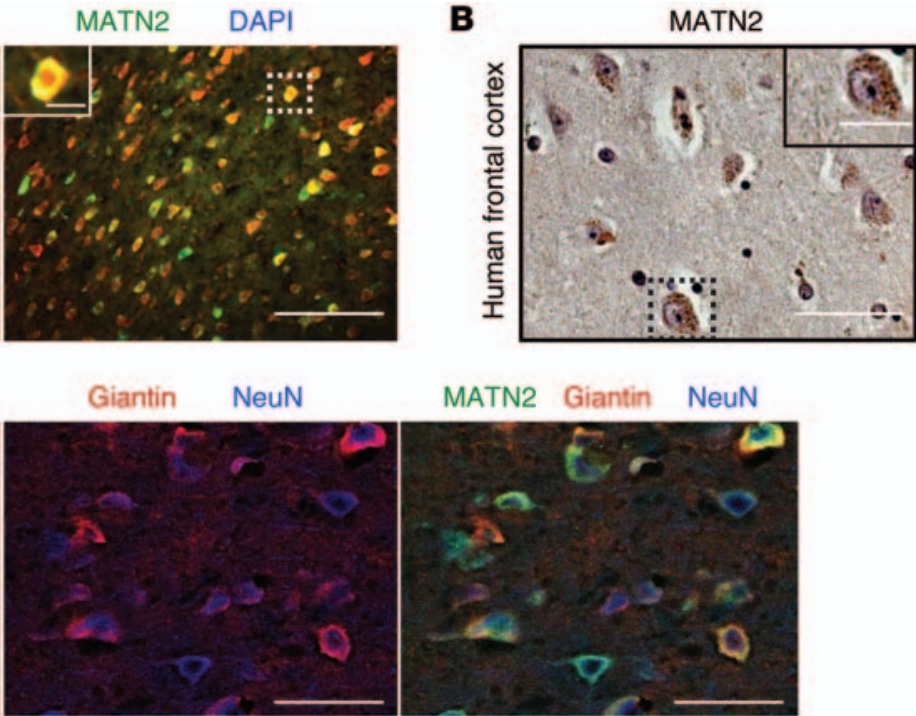

EAE frontal cortex

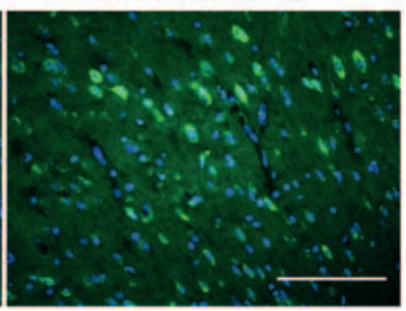

E

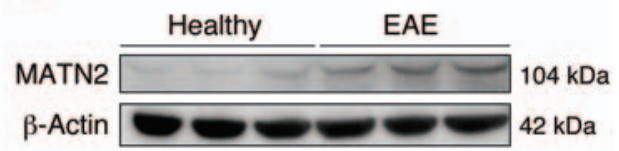

\section{$\mathbf{F}$}

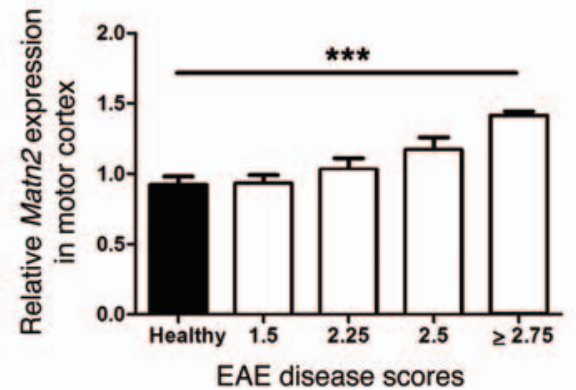

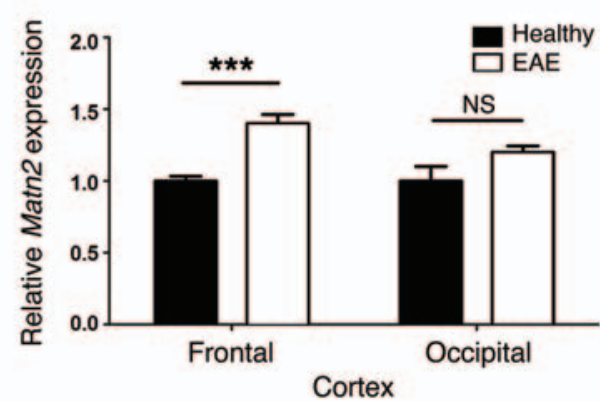

Figure 2. MATN2 is expressed in neurons and is increased in EAE motor cortex. (A) Labeling for MATN2 or colabeling for MATN2 and NeuN (pan-neuronal nuclear marker) in the frontal cortices of healthy mice. Scale bar: $100 \mu \mathrm{m}$ (left); $50 \mu \mathrm{m}$ (right); $12.5 \mu \mathrm{m}$ (inset). (B) MATN2 IHC labeling in the human frontal cortex. Scale bar: $50 \mu \mathrm{m} ; 25 \mu \mathrm{m}$ (inset). (C) Confocal images showing colabeling for MATN2, NeuN, and giantin in the frontal cortices of healthy mice. Scale bar: $10 \mu \mathrm{m}$. (D) Labeling for MATN2 in the frontal cortices of healthy mice and EAE mice. Scale bar: $50 \mu \mathrm{m}$. (E) Denaturing immunoblot analysis reveals increase in MATN2 protein in the frontal cortices of EAE mice (day 21, grade 3) compared with healthy controls. (F) Matn2 gene expression (mean \pm SEM, qRT-PCR) in the motor cortices of EAE mice (day 21) is associated with disease score and specific for the frontal cortex region ( $n=4-6$ per group).

${ }^{* * *} P<0.001,1$-way ANOVA/2-way ANOVA.

transcriptional regulation using quantitative real-time PCR (qRT-PCR) in an independent cohort of mice (Figure 1C). One of the most highly induced genes was Matn2 ( $P=0.00002$, fold change $=1.7$ ). Since MATN2 has been shown recently to play a role in peripheral nerve regeneration after crush injury (22), we selected this molecule for further investigation.

MATN2 is expressed in neurons of mouse and human brains and is increased in acute EAE motor cortices. The gene structure of mouse and human Matn 2 show a 93\% similarity, $86.5 \%$ identity (25), and an identical basic gene structure (26), and previous studies have detected MATN2 in the murine CNS $(27,28)$. How- ever, a cell-specific expression analysis has not been performed to date. Using immunofluorescence labeling, we found that, under physiological conditions, MATN2 protein was expressed by NeuN-positive neurons (Figure 2A), particularly in cortical layers $2 / 3$ and 5 (Supplemental Figure 1A), in which corticospinal motoneurons are located. In addition, MATN2 was highly expressed by hippocampal neurons (Supplemental Figure 1B). This was consistent with Matn2 expression levels in primary murine CNS cell monocultures, in which we found that neurons expressed the highest levels of Matn2 compared with various glial cells (Supplemental Figure 1C). 
A
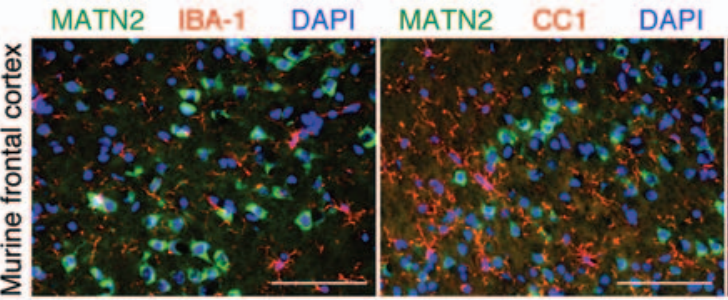

MATN

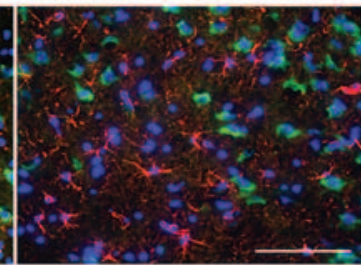

B

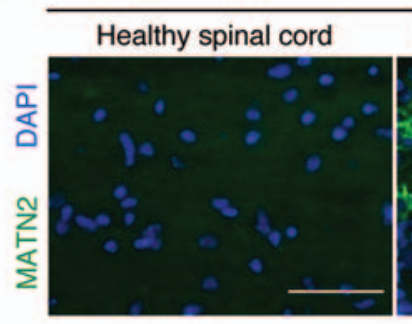

Murine EAE
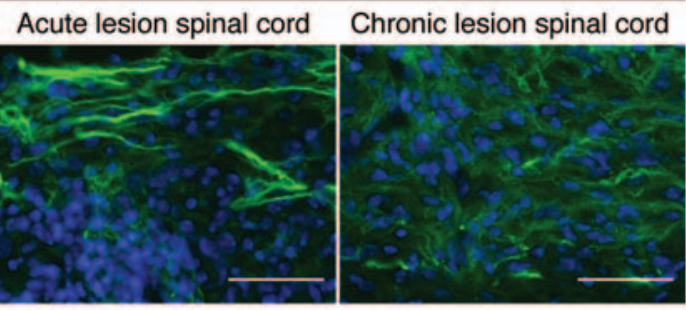

C

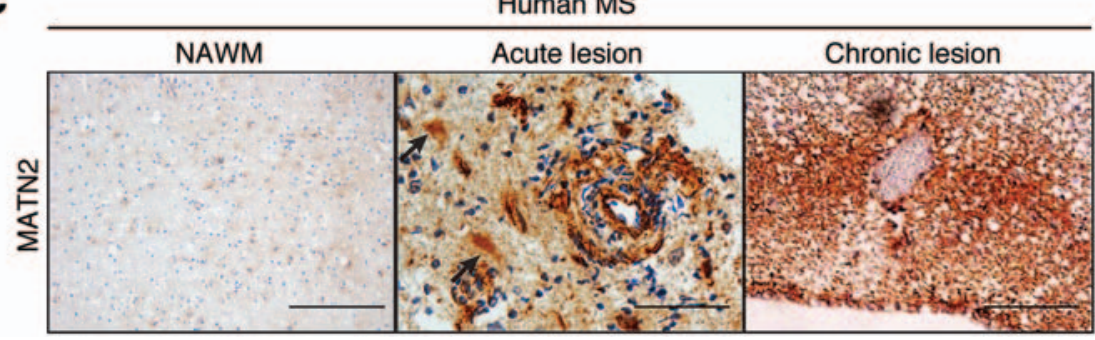

Figure 3. MATN2 expression is specific to neurons in nonlesioned EAE cortex but differentially expressed in murine EAE and human MS lesions. (A) Colabeling for MATN2 and IBA-1, CC1, or GFAP in the frontal cortices of healthy mice. Scale bar: $50 \mu \mathrm{m}$. (B) Labeling for MATN2 on longitudinal spinal cord sections of healthy, acute, and chronic EAE lesions. Scale bar: $12.5 \mu \mathrm{m}$. (C) IHC labeling for MATN2 in normal-appearing white matter (NAWM) and early acute and chronic human MS lesions. Acute lesions show lesional extracellular, astrocytic (black arrows), and perivascular expression of MATN2. Scale bar: $200 \mu \mathrm{m}$ (NAWM); $50 \mu \mathrm{m}$ (acute); $100 \mu \mathrm{m}$ (chronic).
Human postmortem tissue revealed MATN2 expression in some cortical and hippocampal neurons, mirroring the murine expression pattern (Figure 2B and Supplemental Figure 1B). Investigation of the neuron-specific location of MATN2 using confocal imaging revealed that MATN2 colocalized with giantin, a marker for the Golgi apparatus in the neuronal cytoplasm (Figure 2C). We next investigated whether MATN2 expression correlated with EAE disease severity and found that both MATN2 protein (Figure 2, D and $\mathrm{E}$ ) and gene expression (Figure 2F) were upregulated in the motor cortex, with a maximal upregulation of 1.7-fold $(P=0.0002$; EAE score $\geq 2.75$, day 21). We further showed that Matn2 expression was not regulated in the EAE mouse occipital cortex, a region without direct neuronal spinal cord projections (Figure 2F).

MATN2 expression is increased in murine EAE and human MS lesions. Colocalization studies with markers for astrocytes, macrophages/microglia, and oligodendroglia showed that MATN2 was expressed within neurons of the nonlesioned cortex (Figure 3A). In the lesion-associated spinal cords of EAE mice, however, we demonstrated that MATN2 protein was differentially expressed. We did not detect MATN2 in healthy spinal cord, but it was present in acute and chronic EAE spinal cord lesions (day 18-21 and day 45-50, respectively; Figure 3B). We detected MATN2 both in association with axons and in the extracellular space at day 18 to 21 but showed that it is predominantly extracellular in its localization with lesion development (day 45-50). Extracellular MATN2 protein expression was both lesional and perilesional, highest in mice with severe disease, and less intense in mice with lower EAE disease scores and fewer lesions (data not shown), confirming an association between the extent of MATN2 accumulation and disease severity.
At later stages of lesion evolution (chronic EAE), extracellular MATN2 became increasingly abundant in spinal cord lesions (Supplemental Figure 2A; $P=0.004$ ) and colocalized with the ECM molecules laminin and fibronectin in perilesional areas (Supplemental Figure 2B), suggesting an association of MATN2 with other disease-induced ECM components.

We also assessed whether MATN2 was expressed in brain lesions of patients with MS. In normal-appearing white matter little or no MATN2 expression was found (Figure 3C). Concordant with the murine MATN2 expression, early acute demyelinating lesions obtained from biopsies showed abundant extracellular MATN2, which was located in perivascular lesion areas but was also present in morphologically identifiable astrocytes (Figure 3C). In chronic MS lesions, MATN2 showed a prominent extracellular location (Figure 3C). It is important to note that biopsied "acute" MS lesions typically evolve over weeks to months after large lesions become symptomatic, whereas acute EAE tissue is sampled within hours/days of true lesion onset, limiting direct comparisons between human and murine lesions.

MATN2 is released by damaged axons in acute EAE spinal cord lesions. Since cortical MATN2 expression is limited to neurons in our mouse model (Figure 2), we hypothesized that projecting axons are the primary source of MATN2 in the acutely injured spinal cord. Using immunofluorescence labeling, we showed a direct colocalization of the axon markers neurofilament and synaptophysin with MATN2 in acute EAE lesions (Figure 4A). In addition, orthogonal confocal imaging of specific axons revealed an intraaxonal location of MATN2 (Figure 4B). 
A

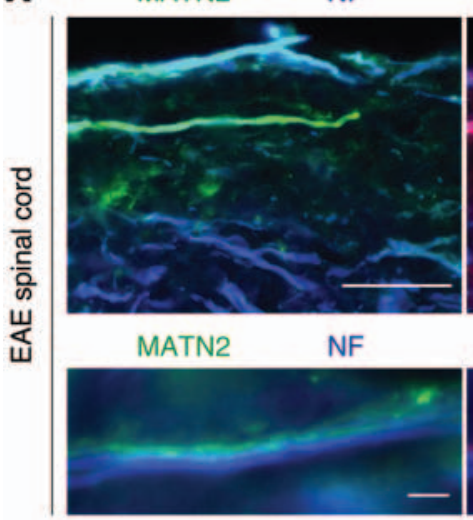

B

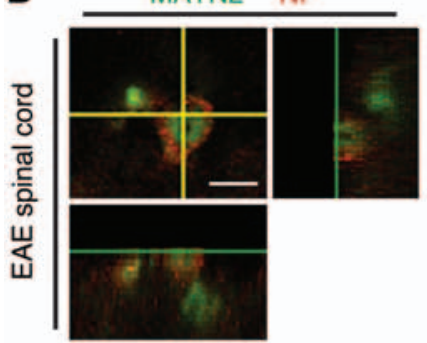

C

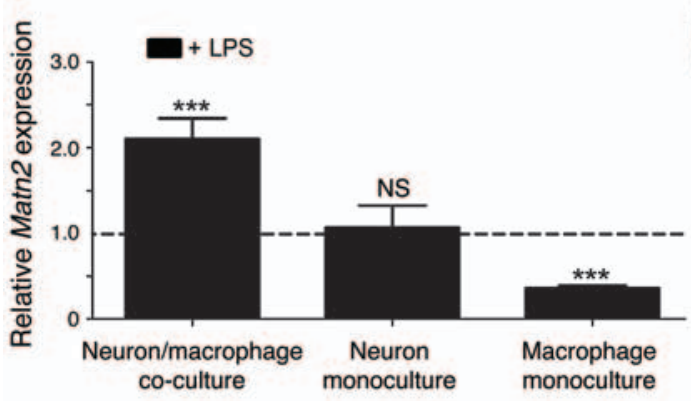

E
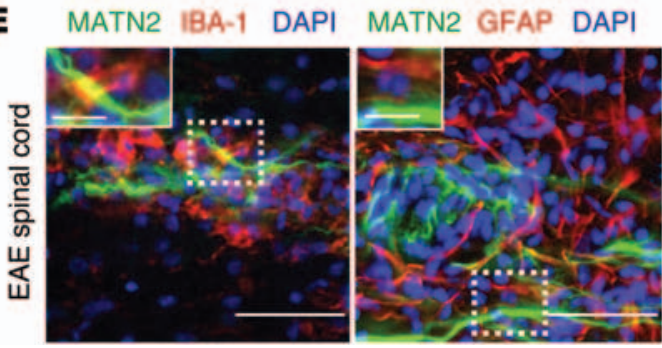

D MATN2

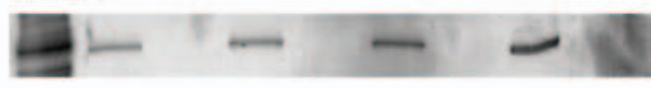

Amido black
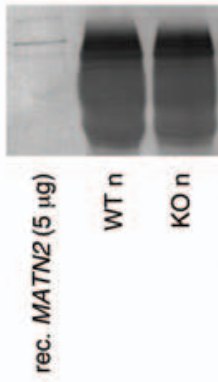

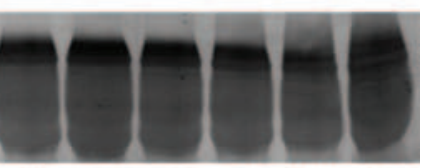

$\begin{array}{ll}\infty & 0 \\ 0 & 0 \\ + & + \\ 5 & 5 \\ 5 & 0 \\ 3 & 8\end{array}$

$\begin{array}{ccc}\infty & E & E \\ 1 & 5 & 5 \\ + & \vdots & \vdots \\ 5 & + & + \\ 0 & 5 & 5 \\ \square & 5 & 0\end{array}$

$\begin{array}{ll}\infty & \infty \\ \vdots & \vdots \\ + & + \\ E & E \\ 5 & 5 \\ 5 & + \\ + & + \\ 5 & c \\ 5 & 0\end{array}$

MATN2 Synaptophysin NF

MATN2 Synaptophysin NF

$-$
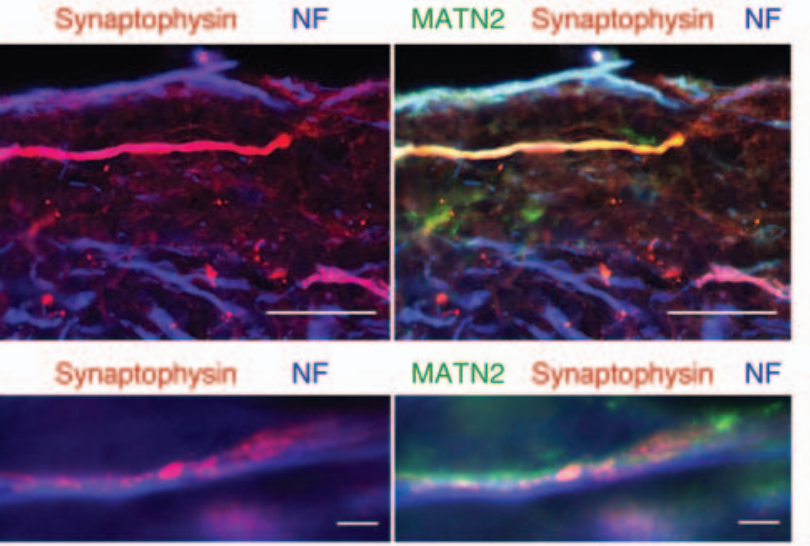

Figure 4. MATN2 is released from neurons following axonal injury in spinal cord lesions. (A) Colabeling for MATN2, synaptophysin, and pan-neurofilament (NF) (top) in acute spinal cord lesions showing clear intraaxonal as well as extraax onal MATN2 expression. Scale bar: $12.5 \mu \mathrm{m}$ (top); $1.5 \mu \mathrm{m}$ (bottom). (B) Confocal 3D image showing colabeling for MATN2 and neurofilament (NF) in an acute spinal cord lesion. Scale bar: $1.5 \mu \mathrm{m}$. (C) Matn2 expression (mean $\pm \mathrm{SEM}$, qRT-PCR) in mono- and cocultures following 24-hour LPS addition shows increased Matn2 expression in LPS-treated cocultures ( $n=6-9$ per group). Gene expression is shown relative to untreated cultures. ${ }^{* *} P<0.001, P \geq 0.5=$ not significant, 2-way ANOVA. (D) Immunoblot analysis reveals specific MATN2 protein expression in WT neuron (WT n) cultures compared with KO controls (KO $\mathrm{n}$ ) and is increased in cocultures following LPS addition (24 hours). WT $\mathrm{m}$, WT macrophage; rec. MATN2, recombinant MATN2.

(E) Colabeling for MATN2 and IBA-1 or GFAP, RIP, or CD3 in acute spinal cord lesions demonstrates close association of MATN2 with macrophages (inset). No colocalization was detected. Nuclei were visualized with DAPI. Scale bar: $10 \mu \mathrm{m} ; 2.5 \mu \mathrm{m}$ (inset).

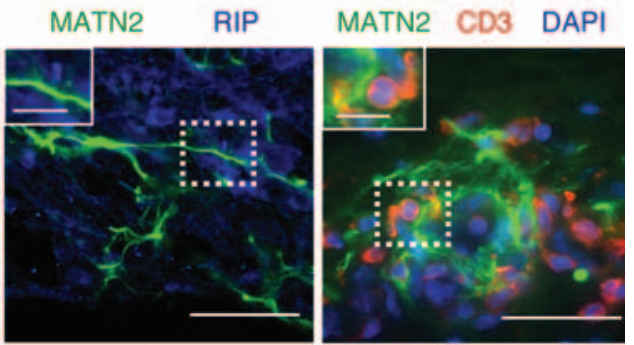

To further investigate our hypothesis that acute inflammatory injury results in the induction of neuronal Matn2, we modified an in vitro model of inflammatory neuroaxonal injury, using a coculture of bone marrow-derived murine macrophages and cortical neurons. In this coculture, LPS addition induced neurite injury within 24 hours, including beading and transection (Supplemental Figure 3A). This was associated with a significant reduction in both neurite length and branching in comparison to untreated control cultures $(P<0.001$ for each comparison; Supplemental Figure 3B) and a significant decrease in cell viability $(P=0.001$; Supplemental Figure $3 C)$, demonstrating the presence of macrophage-induced neuronal and axonal injury in this model. Using this coculture system, we assessed Matn 2 expression and showed a significant increase in Matn $2 \mathrm{mRNA}$ in the cocultures after LPS addition, relative to untreated cocultures $(P=0.0001 ;$ Figure $4 \mathrm{C})$. In contrast, Matn2 expression was decreased in primary macrophage monocultures after LPS addition $(P=0.0002)$ and unchanged in primary neuronal cultures.

To directly demonstrate neuronal MATN2 protein secretion following axonal injury, we assayed for MATN2 in the supernatant of neuron-macrophage cocultures using neurons from WT or Matn2 KO mice. We found that MATN2 protein could only be detected in the supernatant of cocultures using WT neurons (Figure 4D), 

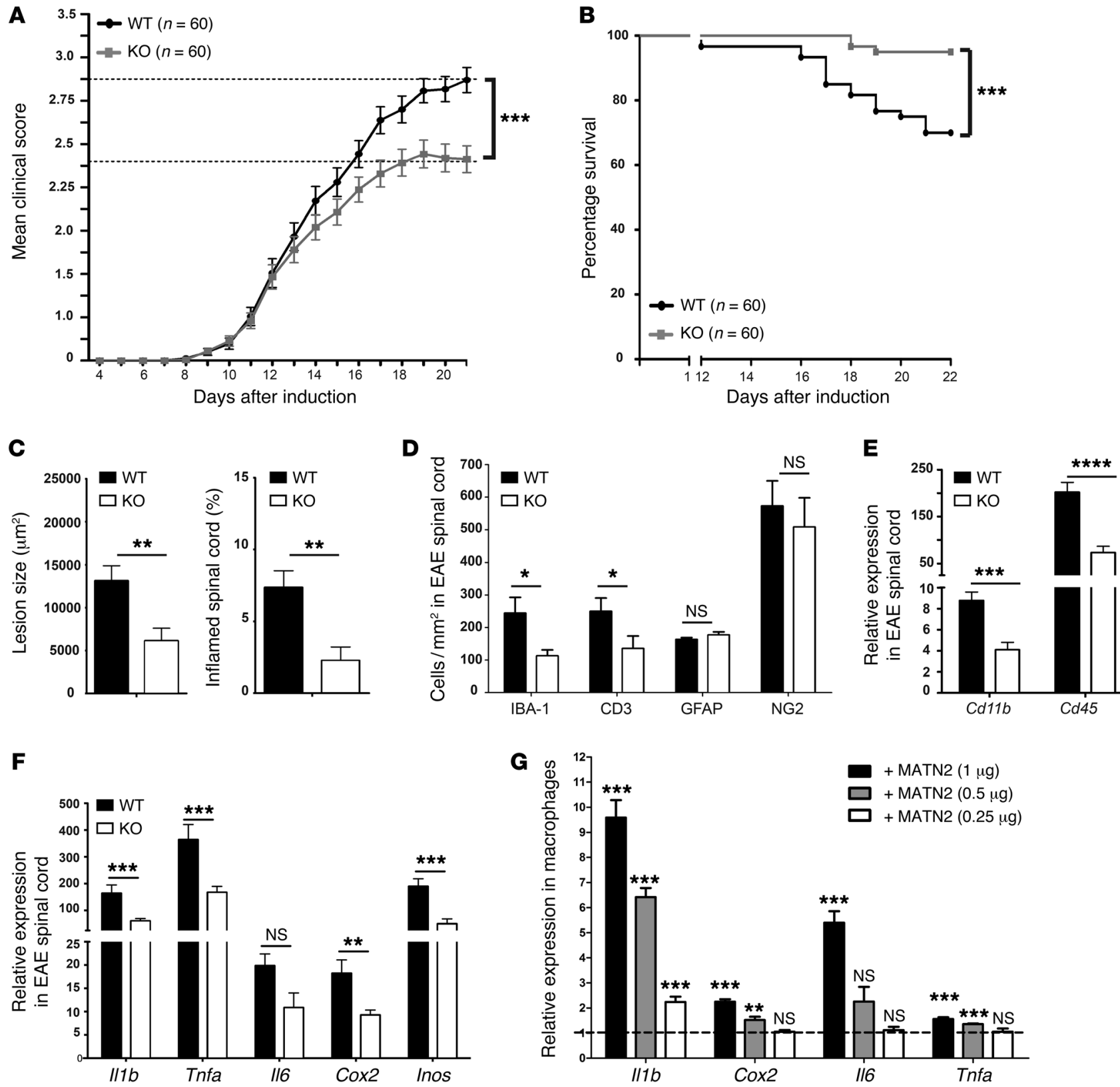

Figure 5. Matn2 deletion reduces EAE disease severity and proinflammatory gene expression in vivo and in vitro. (A) Significantly reduced disease severity (EAE grades, mean \pm SEM) at day 21 in Matn2 KO mice compared with WT mice. Data presented are averaged from 4 independent cohorts with 20 to 40 mice and a total number of 60 mice per genotype. All 4 cohorts showed significantly reduced disease severity in Matn $2 \mathrm{KO}$ mice. ${ }^{* * *} P<0.001$, Mann-Whitney rank-sum test. (B) Survival of EAE-induced WT and Matn2 KO mice (days 0-22) shows significantly greater survival of Matn2 KO mice ( $n=60$ per genotype). ${ }^{* *} P<0.001$, log-rank test. (C) Comparison of lesion size and cross-sectional cord area (\%) with inflammatory lesions in the lumbar spinal cords (mean \pm SEM) in Matn2 KO and WT mice in acute EAE ( $n=10$ per genotype). ${ }^{* *} P<0.01$, Student's $t$ test. (D) Quantification of IBA-1-positive macrophages, CD3-positive T cells, NG2-positive oligodendroglia, and GFAP-positive astrocyte density (mean \pm SEM) in the lumbar spinal cords of WT and Matn2 KO mice (acute EAE) ( $n=10$ per cohort). ${ }^{*} P<0.05$, Student's $t$ test. (E and $\mathbf{F}$ ) Expression of inflammation-associated genes (mean \pm SEM, qRTPCR) in whole spinal cords from acute EAE WT and Matn2 KO mice ( $n=4-6$ per group). ${ }^{* * *} P<0.0001,{ }^{* * *} P<0.001,{ }^{* *} P<0.01, P \geq 0.5=$ not significant, Student's $t$ test. (G) Dose response of induced expression (mean \pm SEM) of inflammation-associated cytokine gene transcripts in cultured bone marrowderived macrophages after addition of recombinant MATN2 for 24 hours ( $n=3$ cultures per condition, 3 independent experiments). Results are shown relative to untreated controls (dotted line). ${ }^{* *} P<0.001,{ }^{* *} P<0.01, P \geq 0.5=$ not significant), 1-way ANOVA.

clearly identifying neurons, rather than macrophages, as the source of MATN2 in this in vitro model. The presence of some MATN2 in all WT cultures could be the result of basal cell death in the first 24 hours of the culture. However, LPS-induced axonal injury increased MATN2 protein levels in the supernatant (Figure 4D). These results, together with the lack of appearance of extracellular MATN2 in the cortices of EAE mice due to the absence of lesions in this area, support our hypothesis that MATN2 may be released from axons or neurons following acute inflammatory injury, either by passive diffusion or by active secretion. 
A
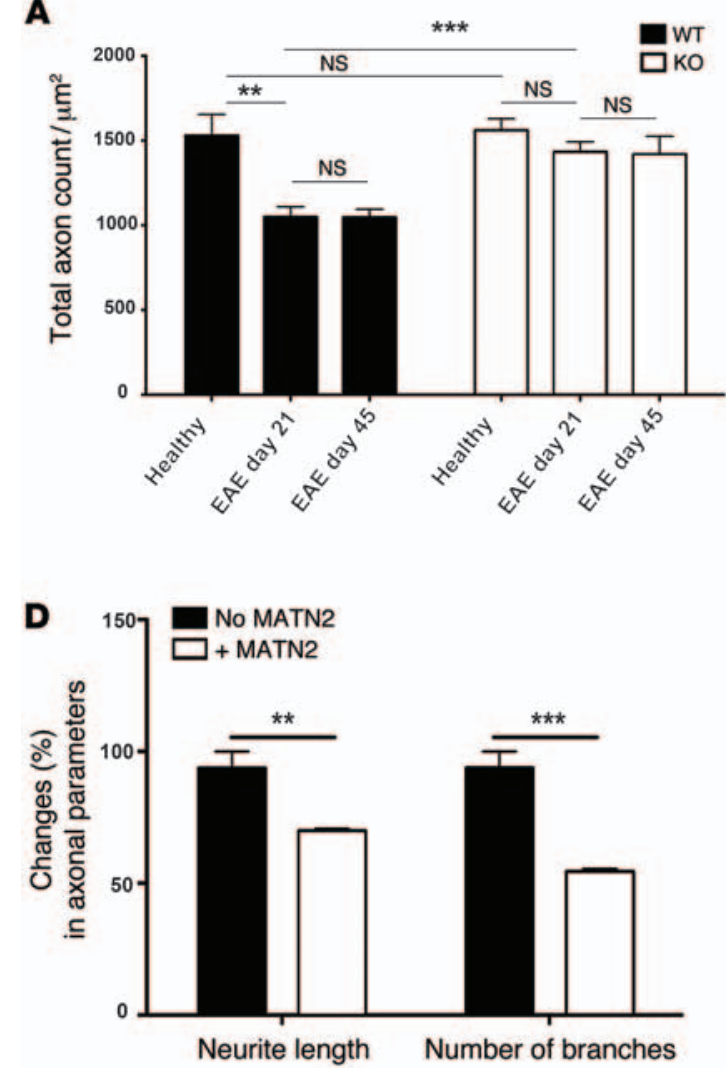

B

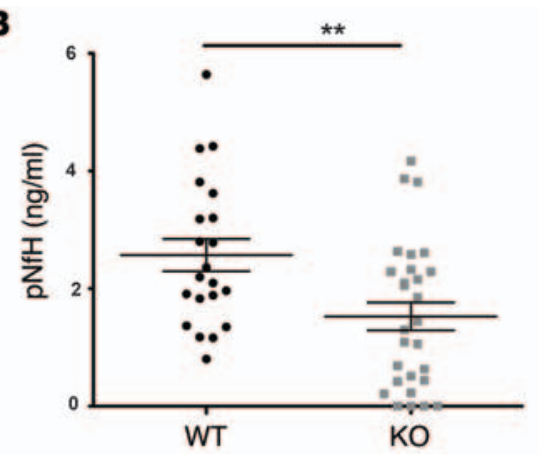

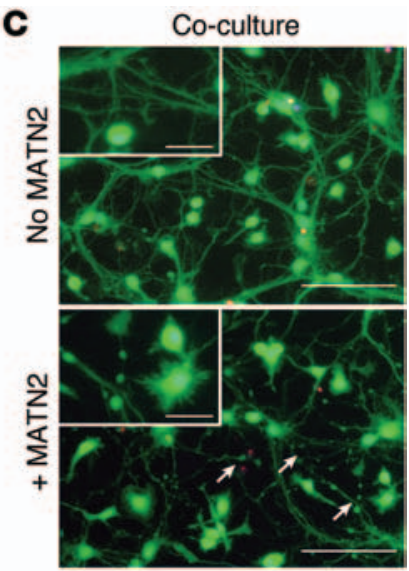

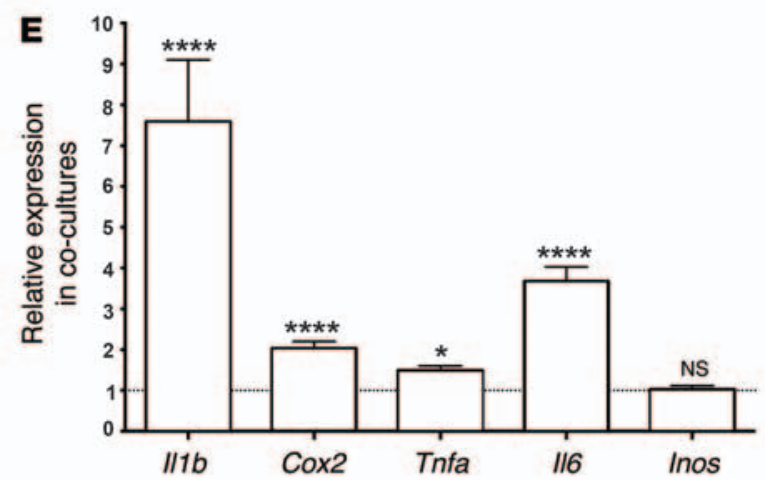

Figure 6. MATN2 contributes to neuroaxonal injury. (A) Axon counts (mean \pm SEM) in the dorsal spinal cord columns of healthy and EAE WT and Matn2 KO mice at day 21 and 45 are reduced in EAE WT mice but not in EAE KO mice $\left(n=4-6\right.$ per group). ${ }^{* * *} P<0.001,{ }^{* *} P<0.01, P \geq 0.5=$ not significant, 2 -way ANOVA. (B) pNfH levels (mean \pm SEM; ELISA) as a marker of axonal injury in sera of WT and Matn2 KO mice at EAE day 21 ( $n=21-26$ per genotype). ${ }^{* *} P<0.01$, Student's $t$ test. (C) Neuron/macrophage cocultures stained with Calcein AM and Ethidium Homodimer after 24-hour incubation with recombinant MATN2 $(1 \mu \mathrm{g} / \mathrm{ml})$ or untreated controls. Arrows indicate neurite beading and transection in the presence of MATN2. Scale bar: $50 \mu \mathrm{m}$; $12.5 \mu \mathrm{m}$ (inset). (D) Assessment of neurite length and number of branches (mean \pm SEM; percentage) of 100 neurons in coculture after 24-hour incubation with recombinant MATN2 (1 $\mu$ g/ml) ( $n=3$ cultures per condition, 3 independent experiments). ${ }^{* * *} P<0.001 ;{ }^{* *} P<0.01$, Student's $t$ test. (E) Expression of proinflammatory cytokines (mean \pm SEM, qRT-PCR) in WT macrophage/KO neuron cocultures following a 24-hour incubation with recombinant MATN2 ( $1 \mu \mathrm{g} / \mathrm{ml})(n=2-4$ cultures per condition, 3 independent experiments). Results are shown relative to untreated controls (dotted line). ${ }^{* * *} P<0.0001 ;{ }^{*} P<0.05, P \geq 0.5=$ not significant, Student's $t$ test.

Although extracellular MATN2 was located in close proximity to infiltrating mononuclear cells such as macrophages and T cells (Figure 4E), MATN2 staining did not colocalize with macrophages, astrocytes, oligodendroglia, or T cells in acute EAE spinal cord, thus excluding these cell types as a source of MATN2 during the early phase of lesion development. Interestingly, MATN2 was present in GFAP-positive astrocytes, CC1-positive oligodendrocytes, and NeuN-positive dorsal horn neurons at chronic disease stages (EAE day 45-50; Supplemental Figure 4A). Consistent with the induction of MATN2 in these other cell types, Matn2 expression at EAE day 50 was markedly increased in the spinal cord, whereas expression in the motor cortex had returned to basal expression levels (Supplemental Figure 4B). Concordant with the EAE expression patterns, we also found intracellular MATN2 expression in cells with astrocytic, oligodendrocytic, and neuronal morphology in chronic MS lesions (Supplemental Figure 4C).

Ablation of Matn2 reduces EAE disease severity and lesion development. Previous studies have shown that deletion of Matn2 in mice causes no obvious phenotypic abnormalities with respect to organ histology, life span, and fertility (25). First, we confirmed that Matn2 deletion occurred in the CNSs of the Matn2 $\mathrm{KO}$ mice at the transcript and protein level (Supplemental Figure 5, A-C) and further showed that, under physiological conditions, cell and axon numbers in the dorsal column of the spinal cords were not different compared to those of WT littermates (Supplemental Figure 5, $\mathrm{D}$ and $\mathrm{E}$ ). We next induced $\mathrm{MOG}_{35-55} \mathrm{EAE}$ in Matn2 $\mathrm{KO}$ mice and WT littermates. The data presented are averaged from 4 independent cohorts (20-40 mice in each cohort), with a total number of 60 mice per genotype (Figure 5A). We found that Matn $2 \mathrm{KO}$ mice had significantly lower disease scores in acute EAE from day 17 $(P=0.01)$ to day $21(P=0.0001$; Figure $5 \mathrm{~A})$, and this effect was apparent within the individual cohorts as well as the grouped data. The mean disease severity in Matn2 KO mice was characterized by mild hind limb weakness, with paresis of the tail (EAE grade 2.25), whereas in WT mice, it comprised complete hind limb and tail paralysis (EAE grade 3). In addition, KO mice showed a significantly higher survival rate ( $P=0.0003$; Figure $5 \mathrm{~B})$.

Lesion assessment of mice with acute EAE (day 18-21) showed that Matn2 KO mice had significantly smaller lesions compared with WT mice $(P=0.008$; Figure $5 \mathrm{C})$, although the density of cells within lesions was similar $(P=0.17$; Supplemental Figure 6A). Overall, a lower percentage of total spinal cord area 


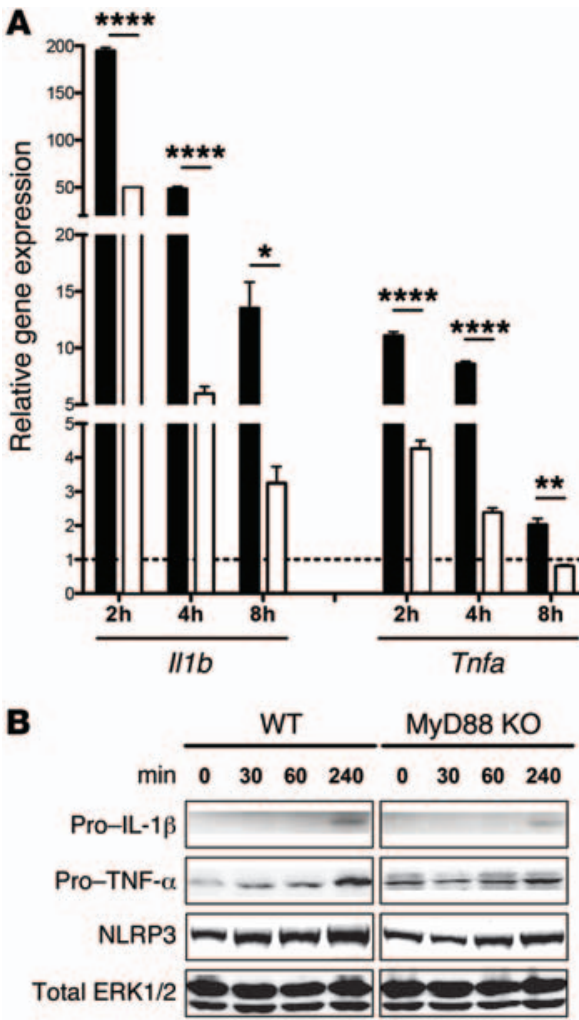

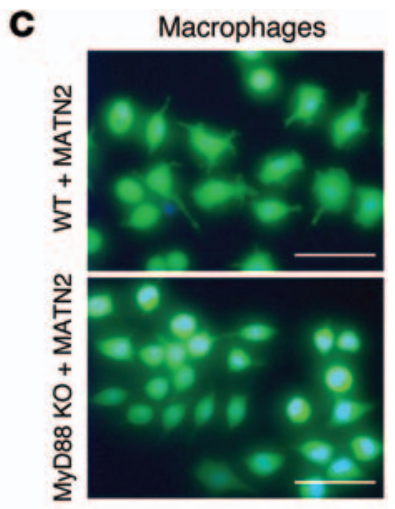

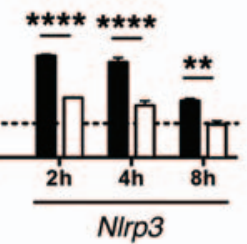

D
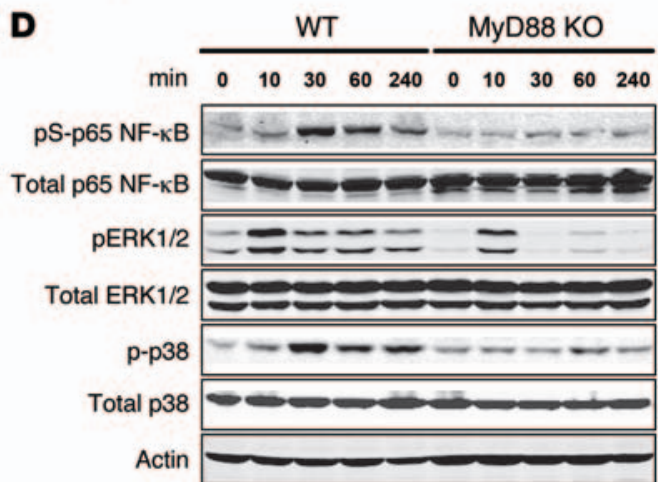

Figure 7. Exogenous MATN2 induces proinflammatory gene expression in macrophages via TLR signaling pathways. (A) Expression of $/ 17 b$, Tnfa, and Nlrp3 in WT and Myd88 KO macrophages (mean \pm SEM, qRT-PCR) following incubation with recombinant MATN2 $(2 \mu \mathrm{g} / \mathrm{ml})$ at indicated time points ( $n=3$ cultures per condition, 3 independent experiments). Results are shown relative to untreated controls (dotted line; ${ }^{* * * *} P<0.0001,{ }^{* *} P<0.01,{ }^{*} P<0.05$ 2-way ANOVA). (B) Immunoblot analysis of whole cell lysates for proIL-1 $\beta$, pro-TNF- $\alpha$, and NLRP3 of WT and MyD88 KO macrophages without or after 30,60 , and 240 minutes of MATN2 incubation. Data show 1 of 4 independent experiments with total ERK as loading control. (C) WT and MyD88 KO macrophage cultures (stained with Calcein AM) after 24-hour incubation with recombinant MATN2 $(2 \mu \mathrm{g} / \mathrm{ml})$. Note that only WT cells are activated following MATN2 addition. Scale bar: $25 \mu \mathrm{m}$. (D) Immunoblot analysis for phosphorylated NF- $\kappa B, p 38$, and ERK $1 / 2$ as well as total NF-kB, ERK1/2, p38, and actin of WT and Myd88 KO macrophage whole cell lysates untreated or after $10,30,60$, and 240 minutes of MATN2 incubation. Data show 1 of 4 independent experiments. was composed of densely packed lesional nuclear cell infiltrates in $\mathrm{KO}$ mice $(P=0.003$; Figure $5 \mathrm{C})$. In addition, the relative proportions of the two major inflammatory lesional cell types, macrophage/microglia (IBA-1) and T cells (CD3), were significantly decreased in KO mice (Figure 5D). This was no longer the case when mice from different genotypes with identical disease grades were compared (Supplemental Figure 6B), indicating that lack of endogenous MATN2 decreased lesion size without selectively inhibiting the migration of $\mathrm{T}$ cells and/or macrophages into the CNS. The difference in EAE disease grades was maintained up to day 43 after induction $(P=0.004$; Supplemental Figure $6 \mathrm{C})$. However, divergence in EAE scores after day 26 after induction was not observed, consistent with the acute and monophasic nature of this EAE model. Accordingly, lesion assessments in chronic EAE mice (day 45-50) revealed that the differences in lesion size and cell numbers were no longer present (Supplemental Figure 6, D and E). This suggests that, in the EAE model examined, endogenous MATN2 primarily exacerbates the acute inflammatory phase of the disease.

MATN2 activates immune cells and induces proinflammatory gene expression in macrophages. Since other ECM molecules, such as hyaluronan and biglycan, have been shown to stimulate the peripheral inflammatory response via macrophage/microglia activation (29), we hypothesized that MATN2 could similarly act as a proinflammatory molecule. We determined $C d 11 b$ and $C d 45$ gene expression levels in acute EAE spinal cords and found a marked reduction in KO mice compared with WT mice (Cd11b expression: $P=0.003, C d 45$ expression: $P=0.0003$; Figure $5 \mathrm{E}$ ). Interestingly, this effect was seen even in WT and KO EAE mice with matched disease severity grades ( $C d 11 b$ expression: $P=0.04$, $C d 45$ expression: $P=0.001$; Supplemental Figure $6 \mathrm{~F}$ ), consistent with a reduced inflammatory activation state of macrophages/ microglia in Matn2 KO mice compared with that in WT mice. To further investigate the effect of MATN2 on macrophages/microglia, we assessed gene expression of proinflammatory molecules and found a marked reduction in Illb, Tnfa, Cox2, and Inos expression in Matn2 $\mathrm{KO}$ mice (Illb expression: $P=0.01$, Tnfa expression: $P=0.03$, Cox 2 expression: $P=0.004$, Inos expression: $P=0.001$; Figure 5F). Again, gene expression changes were still significantly reduced in selected mice from both genotypes with matched disease grades (Il1b expression: $P=0.01$, Tnfa expression: $P=0.03$, Cox2 expression: $P=0.02$, Inos expression: $P=0.001$; Supplemental Figure 6G). These results suggest that MATN2 directly or indirectly upregulates proinflammatory molecule expression during acute EAE-associated neuroinflammation.

To further determine whether MATN2 can induce proinflammatory gene expression in macrophages, we added recombinant MATN2 to cultured murine bone marrow-derived macrophages and examined gene expression changes 24 hours later. Consistent with our in vivo data, we found a dose-dependent increase in expression of Il1b (maximum $P=0.00008$ ), Cox2 (maximum $P=0.0001$ ), Il6 (maximum $P=0.0001$ ), and Tnfa (maximum 


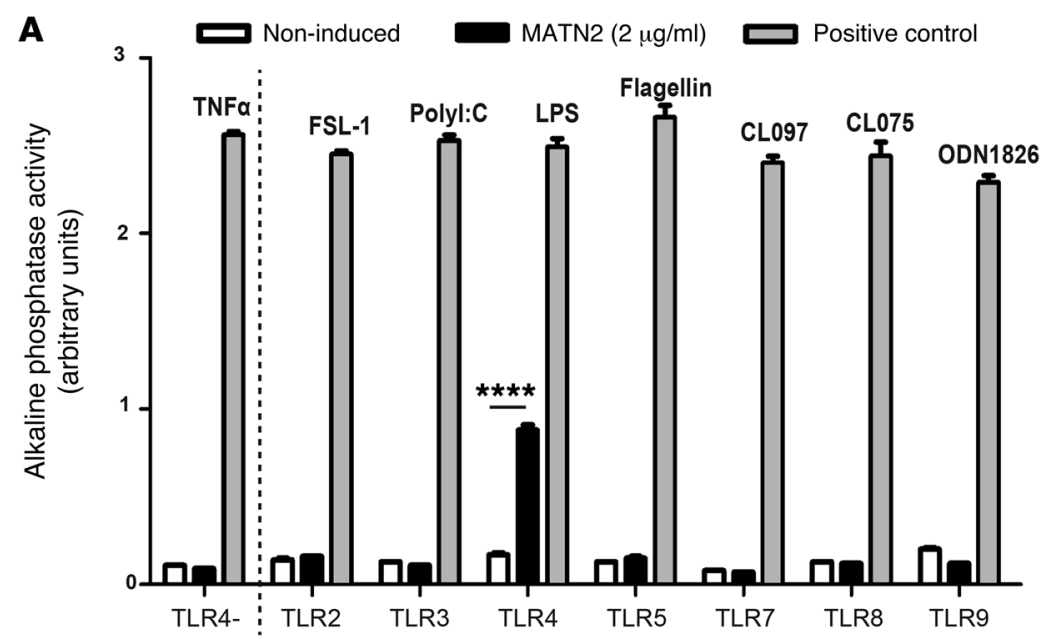

B

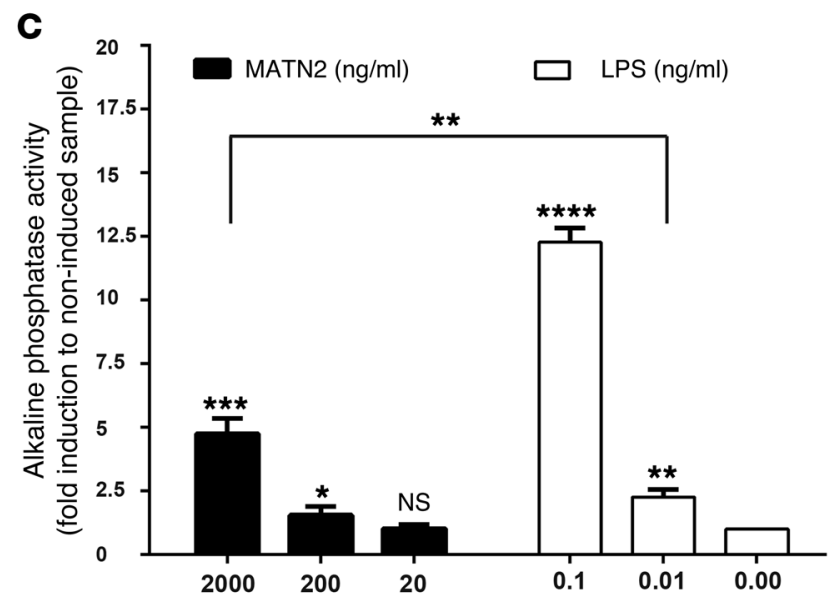

Figure 8. MATN2 signals through TLR 4. (A) Screening for MATN2-triggered ( $2 \mu \mathrm{g} / \mathrm{ml})$ activation of the alkaline phosphatase reporter via TLR/NF- $\mathrm{B}$ signaling in genetically modified HEK293 cells expressing the indicated type of TLR (black bars). Alkaline phosphatase activity (arbitrary units) is used as an indicator for TLR stimulation. As positive control, cell lines were induced with a receptor-specific ligand. TLR2, heat-killed Listeria monocytogenes at 108 cells/ml; TLR3, poly(I:C) at $1 \mu \mathrm{g} / \mathrm{ml}$; TLR4, E. coli K12 LPS at $100 \mathrm{ng} / \mathrm{ml}$; TLR5, S. typhimurium flagellin at $100 \mathrm{ng} / \mathrm{ml}$; TLR7, CL097 at $1 \mu \mathrm{g} /$ $\mathrm{ml}$; TLR8, CL075 at $10 \mu \mathrm{g} / \mathrm{ml}$ + poly(dT) $10 \mu \mathrm{M}$; and TLR9, CpG ODN 1826 at $100 \mathrm{ng} / \mathrm{ml}$. Noninduced TLR clones were used as negative controls (white bars), and a HEK293 cell line expressing only alkaline phosphatase but not TLR4 was used as specific TLR4 negative control (TLR4-). (B and C) Fold induction in alkaline phosphatase triggered by (B) $2 \mu \mathrm{g} / \mathrm{ml}$ MATN2 or $100 \mathrm{ng} / \mathrm{ml} \mathrm{LPS} \mathrm{or} \mathrm{by} \mathrm{(C)} \mathrm{the} \mathrm{indicated} \mathrm{doses} \mathrm{of} \mathrm{MATN2} \mathrm{or} \mathrm{LPS.} \mathrm{Note} \mathrm{that} \mathrm{the}$ response elicited by $2,000 \mathrm{ng} / \mathrm{ml}$ MATN2 is significantly greater than that of the trace LPS concentration of $0.01 \mathrm{ng} / \mathrm{ml}(P=0.003)$. ${ }^{* * *} P<0.0001$, ${ }^{* * *} P<0.001,{ }^{* *} P<0.01,{ }^{*} P<0.05$.

$P=0.0001$ ) (Figure 5G), indicating that MATN2 can directly induce proinflammatory gene expression in macrophages.

MATN2 contributes to neuroaxonal injury. To determine whether MATN2 has an effect on neuroaxonal injury, we analyzed axon numbers in the dorsal columns of healthy and EAE WT and Matn2 KO mice. EAE WT mice showed a significant loss of axons relative to WT controls $(P=0.003)$, with marked inflammatory cell infiltration and abnormally shaped axons (Figure 6 and Supplemental Figure 7). In contrast, EAE in KO mice did not result in axonal loss compared with that in Matn2 KO controls $(P=0.17)$, and Matn2 KO EAE mice had a significantly higher axon density than WT EAE mice at day $21(P=0.0002$; Figure 6A and Supplemental Figure 7). Although more fibrous ECM tissue accumulated visually in the WT spinal cord in chronic EAE (day 45) (Supplemental Figure 7), no additional axon loss was found in mice of either genotype ( $P=0.9$ for both; Figure $6 \mathrm{~A})$, indicating that axonal loss in our EAE model is confined to the acute stage of the disease and that no on-going deleterious effect of MATN2 on axon integrity occurred up to 45 days after disease induction. Reduced axonal injury in Matn2 KO mice with acute EAE was further confirmed by lower levels of circulating phosphorylated neurofilament heavy chain ( $\mathrm{pNfH})$ protein $(P=0.006$; Figure $6 \mathrm{~B})$, a marker that correlates with axonal loss in the dorsal column of EAE mice (6).

To model the potential effect of MATN2-mediated macrophage activation on neurons, we cocultured WT macrophages with cortical neurons from Matn2 KO mice and assessed measures of neuroaxonal injury following addition of recombinant MATN2. After a 24-hour incubation, we observed marked induction of neurite beading and transection in MATN2-treated cultures when compared with untreated controls (Figure 6C). We also detected a significant reduction in total neurite length $(P=0.002)$ and neurite branching $(P=0.001)$ in these cultures (Figure 6D). However, MATN2 addition to neuron monocultures did not induce neuronal injury or cell death (Supplemental 
Figure 8, A and B). Similar to the macrophage monoculture, we also identified increased expression of $\operatorname{Il} 1 b(P=0.0006)$, Cox2 $(P<0.0001)$, Tnfa $(P=0.002)$, and Il6 $(P<0.0001)$ after MATN2 addition in the cocultures (Figure $6 \mathrm{E}$ ). Taken together, these results indicate that MATN2 can induce activation of macrophages, potentially contributing to neuroaxonal injury.

MATN2 signals via TLR signaling pathways in macrophages. Previous studies have shown that several ECM DAMPs activate intracellular signaling pathways via the family of TLRs, particularly TLR2 and TLR4, leading to the induction of proinflammatory cytokines via the adaptor protein myeloid differentiation factor 88 (MyD88) $(9,11,12,15)$. To assess whether MATN2 induces Il-1 $\beta$ and TNF- $\alpha$ through TLR/MyD88-mediated pathways in macrophages, we compared mRNA expression levels in response to exogenous MATN2 addition to WT and Myd88 KO macrophages. Consistent with our previous results showing an increase of both transcripts 24 hours after MATN2 addition (Figure 5E), we also found increased $I l 1 b$ and Tnfa mRNA expression in WT macrophages 2-8 hours following MATN2 addition, with a maximal induction at the 2-hour time point (Il1b expression: $P<0.0001$, Tnfa expression: $P<0.0001$; Figure 7A). By contrast, induction of both transcripts was significantly lower in $M y D 88 \mathrm{KO}$ macrophages at all investigated time points (Il1b expression: $P<0.0001$, Tnfa expression: $P<0.0001$; Figure 7A). Since activation and secretion of pro-IL-1 $\beta$ into its mature form is dependent on an accumulation of NLRP3 protein in the cell (30), we also examined whether stimulation of macrophages with MATN2 leads to the production of NLRP3. Indeed, Nlrp3 mRNA expression was significantly increased in WT macrophages following MATN2 addition $(P<0.0001)$ and showed a markedly lower expression in $M y d 88 \mathrm{KO}$ macrophages $(P<0.0001 ;$ Figure 7A). These results were confirmed by Western blot analysis and showed, 4 hours after MATN2 addition, an increase in pro-IL-1 $\beta, \mathrm{TNF}-\alpha$, and NLRP3 protein levels, which were considerably lower in Myd88 KO macrophages (Figure 7B). Therefore, these results suggest that MATN2 signals at least partially through TLR-mediated signaling pathways. We further observed that MATN2 addition led to changes in the shape of WT macrophages that were consistent with the cells being in an activated state, whereas $\mathrm{KO}$ cells appeared to remain in a resting state (Figure $7 \mathrm{C}$ ).

To identify downstream pathways associated with DAMP/ TLR/MyD88-dependent signaling in response to MATN2, we examined phosphorylation of the MAP kinases ERK and p38 as well as the transcription factor NF- $\kappa \mathrm{B}$. In WT macrophages, we detected a prominent increase in ERK, p38, and NF- $\mathrm{KB}$ (p65) phosphorylation following MATN2 incubation, reaching a maximum after 10 minutes for ERK and after 30 minutes for p38 and NF- $\kappa \mathrm{B}$ (Figure 7D). In contrast, phosphorylation of NF- $\kappa \mathrm{B}$ and p38 remained unchanged compared with baseline levels in Myd88 KO macrophages. However, phosphorylation of ERK was still induced, although with lower intensity and duration. Collectively, these results confirm that MATN2 signaling involves TLR family receptor activation, including signaling via the ERK, p38, and NF- $\kappa$ B pathways, leading to MATN2-dependent induction of pro-IL-1 $\beta$, TNF- $\alpha$, and NLRP3.

MATN2 signals through TLR4. To identify the potential receptor for MATN2, we used HEK293 cells expressing the murine TLR2, TLR3, TLR4, TLR5, TLR7, TLR8, or TLR9 receptors, together with alkaline phosphatase, under the control of the $I 112 p 40$ promoter, which is induced by the activation of NF- $\kappa \mathrm{B}$. In these assays, $2 \mu \mathrm{g} / \mathrm{ml}$ recombinant MATN2 or the appropriate positive control ligand was added to each HEK293 culture. Surprisingly, activation of alkaline phosphatase by MATN2 was detected exclusively in TLR4expressing cells (Figure 8A; $P=0.00001$ ). The magnitude of MATN2-induced alkaline phosphatase activity was approximately one-third of that induced by the TLR4 ligand LPS $(200 \mathrm{ng} / \mathrm{ml}$; Figure 8B). By contrast, in HEK293 cells expressing only the reporter gene but not the TLR4 receptor (TLR4-negative control), alkaline phosphatase was not induced by addition of MATN2 (Figure $8 \mathrm{~A})$. An additional experiment using a range of MATN2 dosages $(2,000,200,20 \mathrm{ng} / \mathrm{ml})$ showed a dose-dependent stimulatory effect on TLR4 at 2,000 ng/ml $(P=0.0005)$ and $200 \mathrm{ng} / \mathrm{ml}$ $(P=0.03)$ (Figure $8 C)$.

As endotoxins (LPS) are major contaminants found in commercially available proteins (31), it is necessary to ensure that TLR4 activation by MATN2 was not solely attributable to the presence of endotoxins within the peptide preparation. According to previous studies showing that 1 endotoxin unit (EU) is equivalent to 100 to $120 \mathrm{pg}$ endotoxin (31), we estimated that the mass of endotoxin contained within $2 \mu \mathrm{g}$ MATN2 (0.031 EU/ $\mu$ g by LAL method; R\&D Systems catalog no. 3234-MN, lot no. NTR026031) would have a maximum contamination of $10 \mathrm{pg}$. To allow direct comparison of trace endotoxin to the observed MATN2-associated stimulatory responses, we investigated induction of alkaline phosphatase using LPS dosages of $10 \mathrm{pg}(0.01 \mathrm{ng} / \mathrm{ml})$ and $100 \mathrm{pg}$ $(0.1 \mathrm{ng} / \mathrm{ml})$. We found that the response elicited by $2 \mu \mathrm{g} / \mathrm{ml}$ MATN2 was significantly greater than that of the trace LPS concentration of $0.01 \mathrm{ng} / \mathrm{ml}$ (Figure $8 \mathrm{C} ; P=0.003$ ), demonstrating that the stimulatory effects of MATN2 are not likely to be entirely attributable to endotoxin contamination.

TLR4 protein and gene expression have been identified in macrophages as well as neurons. In an attempt to investigate whether MATN2 could also exert its effects by autocrine signaling in neurons, we examined gene expression of Tlr 4 in monocultures of both cell types, which revealed a markedly higher gene expression in macrophages than in neurons (Supplemental Figure 9A). After LPS addition to the monocultures, expression of Tlr4 was induced only in macrophages. MATN2 did not change the expression of Tlr4 in either cell type (Supplemental Figure 9B). These results again suggest that the presence of macrophages is required to effect MATN2-induced axonal injury. However, we cannot completely exclude an autocrine-signaling pathway in neurons as contributing to this response.

Collectively, our results demonstrate that MATN2 is a TLR4 ligand that activates $\mathrm{p} 38, \mathrm{ERK}$, and $\mathrm{NF}-\kappa \mathrm{B}$ in macrophages, which leads to the expression of proinflammatory molecules and the likely induction of neuroaxonal injury in coculture systems.

\section{Discussion}

In our initial studies, we used microarray technology to identify several ECM proteins, including MATN2, which are upregulated in the EAE cortex in response to acute inflammation in the spinal cord. Although only a limited number of studies have investigated the functions of ECM molecules in disease, there is strong evidence to suggest that some of them can act as danger signals, 
so-called DAMPs $(9,13,14,30,32)$, which are released from cells following tissue stress or injury. DAMPs are recognized by innate immunity receptors and, functioning in a similar way to pathogen-associated molecular patterns (33), they induce or increase an acute local proinflammatory response. Our data collectively indicate that MATN2 functions as a neuroaxonally produced DAMP in the CNS.

Histological characterization of MATN2 expression in the acute EAE cortex and spinal cord revealed that MATN2 expression is increased in cortical neurons and also colocalizes to axons within inflammatory spinal cord lesions. Based on these observations, we hypothesized that acute lesional ECM-associated MATN2 originates from cortical neurons and is either actively secreted or passively released from lesional axons. Conversely, at postacute EAE stages and in all MS lesions examined, MATN2 was expressed in lesional and perilesional CNS cells, particularly astrocytes. Thus, it is likely that glial cells contribute to the continued accumulation of MATN2 in EAE and MS lesions, with increasing lesion chronicity. It is possible that ECM-bound MATN2 plays a distinct role at later disease stages; however, as was described previously for the DAMP biglycan (15), our data suggest that the proinflammatory functions of MATN2 are limited to acute inflammatory disease stages when MATN2 is present in its soluble form.

During the postacute disease stage, when inflammation-induced axon injury is no longer present, we did not observe modulation of disease severity (pathologically or molecularly) associated with the presence of ECM-associated MATN2 up to 45 days after EAE induction. However, it remains possible that MATN2 could modulate demyelination or remyelination without affecting EAE severity grades, and this should be investigated using more suitable models in the future.

Underscoring the deleterious role of endogenous MATN2, we showed that Matn2 KO mice subjected to EAE had a significant survival benefit, markedly attenuated acute disease severity, reduced axonal loss, and lower expression of proinflammatory genes compared with WT mice. Interestingly, assessment of cytokine profiles in WT and Matn2 $\mathrm{KO}$ mice matched for EAE grade revealed a persistent reduction in many cytokine expression profiles classically associated with activated macrophages in Matn2 KO mice. Our interpretation of this finding is that, for a given EAE grade, the relative contribution of macrophage activity to this composite end point must be relatively reduced in $\mathrm{KO}$ mice compared with that in WT mice compared with contributions, for instance, from T-lymphocyte cells. This result, therefore, provides further correlative evidence that endogenous MATN2 in EAE activates lesional macrophages.

Using an in vitro model of acute axonal injury, we extended our observations to show that exogenous soluble MATN2 causes production of proinflammatory molecules in macrophages, in particular IL-1 $\beta$. In parallel studies, another matrilin family member, matrilin-3, has also been shown to induce the expression of proinflammatory genes, including Il1b, Tnfa, Il6, Cox2, and Inos, in primary human chondrocytes (18). Interestingly, production of mature IL-1 $\beta$ in macrophages is also induced by other ECM DAMPs, such as biglycan and hyaluronan $(30,34)$. Importantly, these studies revealed that both molecules led to the production of a fully functional and active IL-1 $\beta$ protein by triggering the induction of both
pro-IL-1 $\beta$ and NLRP3 autonomously (30). We were able to show a similar increase in IL-1 $\beta$ and NLRP3 protein and mRNA expression following MATN2 addition. However, the production of fully functional IL-1 $\beta$ in response to MATN2 remains to be confirmed.

Interestingly, ECM components, such as fibronectin extra domain A, hyaluronan-derived oligosaccharides, lumican, and biglycan, are now accepted as endogenous ligands of pattern recognition receptors, in particular TLR2 and TLR4. Signal transduction through TLRs is well characterized, mediated through two possible routes involving the adapter molecules MyD88 and TRIF (35). While signal transduction through most TLRs is solely dependent on MyD88, TLR4 can also signal through an alternative TRIF-dependent mechanism. MyD88-mediated activation of downstream pathways involves phosphorylation of the MAP kinases ERK and p38 as well as the transcription factor NF- $\mathrm{BB}$ $(13,32,36)$. Although most TLR-activating ECM molecules signal through the same TLRs, they all have obvious structural differences and trigger differential responses and biological outcomes. For an example of this, by comparison, biglycan signaling induces proinflammatory TNF- $\alpha$ and pro-IL- $1 \beta$, which in turn induces the expression of COX-2 and IL-6 $(9,13,30)$, whereas decorin promotes the induction of antiinflammatory IL-10 (37). In macrophages, we found increased phosphorylation of NF- $\mathrm{B}$, p38, and ERK following exogenous MATN2 addition, leading to the expression of proinflammatory cytokines. Compared with WT cells, Myd88 KO macrophages had markedly lower IL- $1 \beta$, TNF- $\alpha$, and NLRP3 protein and gene induction and considerably lower induction of NF- $\mathrm{KB}, \mathrm{p} 38$, and ERK1/2 phosphorylation following MATN2 addition. These data suggest that MATN2 acts as a novel ligand for TLR-mediated signaling in macrophages. We were then able to show specific activation of the TLR4 receptor by MATN2 in a HEK cell assay. This specific activation of TLR4 by MATN2 is in line with our previous observation that MATN2-induced proinflammatory gene and protein expression is incompletely inhibited in Myd88 KO cells, as TLR4 can also induce activation through the alternate TRIF-dependent pathway. The presence of MyD88-independent ERK phosphorylation also suggests the involvement of other, as yet, unidentified receptors in MATN2 signaling.

In addition to macrophage-mediated effects, it is also possible that MATN2 could directly signal in axons and neurons in an autocrine fashion, because following tissue injury, TLR4 protein and gene expression is known to be increased in cortical neurons (38-40). In our experiments, exogenous MATN2 added to neuronal monocultures did not induce Tlr4 expression or promote neuronal cell death; however, further work is required to exclude the possibility that MATN2 also signals directly through neurons.

In summary, the present study has identified MATN2 as an important neuronal danger-associated molecule in the CNS that is released in association with tissue damage to elicit a robust proinflammatory response by cells of the innate immune system. We showed that, in the highly aberrant EAE disease state, MATN2 expression in acute inflammatory lesions was associated with worsened disease, likely to be mediated by increased axon loss and more severe acute inflammatory infiltration. In principle, blockade of MATN2 signaling or its neutralization could be a novel therapeutic strategy for early relapsing-remitting MS, in which severe lesional axonal injury is often a feature. 


\section{Methods}

Mice. We used female and male Matn2 KO mice (25) and their WT littermates on a C57BL/6 background. Mice were backcrossed for 10 generations to achieve a $>98 \%$ C57BL/6 background for EAE experiments. $M y d 88 \mathrm{KO}$ mice (41) were previously backcrossed with C57BL/6 mice for over 10 generations.

EAE induction. $\mathrm{MOG}_{35-55}$ EAE was induced in 8- to 12-weekold sex-and age-matched C57BL/6 mice as described previously (42). EAE mice were weighed, monitored, and clinically assessed according to the following grading scale: $0=$ no symptoms; $1=$ distal tail weakness; 1.5 = tail weakness and some hind limb weakness; 2 = complete tail paralysis; 2.25 = complete tail paralysis and gait abnormality; 2.5 = complete tail paralysis and severe gait abnormality; $2.75=$ complete tail paralysis and monoplegia; $3=$ paraplegia; $3.5=$ inability to right when placed on back or significant forelimb weakness; 4 = euthanized or spontaneous death. EAE induction in Matn2 KO mice for analysis of acute disease was performed in 4 independent cohorts of WT and KO littermates, and disease grades were averaged for all mice.

Microarray. Gene expression in motor cortex-enriched tissue was assessed from 4 healthy mice and $6 \mathrm{EAE}$ female mice. Tissue was collected from mice with paraplegia or monoplegia, with contralateral hind limb paresis (EAE grade $\geq 2.75$; day 18-21). For tissue collection, the mice were euthanized and perfused with $20 \mathrm{ml}$ MT-PBS followed by $10 \mathrm{ml}$ RNAlater (Ambion), and forebrains were removed and placed into a cold metal brain matrix. The brains were cut into 1-mm coronal slices, and regions of interest were dissected with the help of a brain atlas (Allen mouse brain atlas; http://mouse.brain-map.org). Region specificity was determined by qRT-PCR analysis for motor cortexspecific genes. RNA was extracted using the RNeasy Mini Tissue Lipid Kit (Qiagen). RNA yield and quality was determined, and processing, including cDNA transcription, labeling and hybridization onto the Affymetrix mouse genome 4302.0 array, and primary quality control measures of the chip results, was performed by the Australian Genome Research Facility (Melbourne, Australia). For biotin labeling of the chip cDNA, $1 \mu \mathrm{g}$ RNA was used. CEL files were analyzed using Partek Genomic suite (version 6.3 beta, Partek). Every GeneChip passed the chip quality control. Lists of significantly changed probe sets ( $P$ values less than 0.05 and fold changes of 1.3 or greater) were generated for each comparison between control and treatment groups. Functional annotation and clustering of the differentially regulated genes were performed using Metacore software (GeneGo, 205 Inc.). The array data is available at the NCBI Gene Expression Omnibus repository (mouse gene expression profiles: GSE47900).

$q R T-P C R$. Tissue was collected as described in the Microarray section. RNA was extracted using the RNeasy Lipid Mini Kit (Qiagen). qRT-PCR was performed on an ABI7700 Sequence Detection System (Applied Biosystems) using the comparative Ct method (43). Primers (GeneWorks) were designed over exon-exon borders, and each primer pair was tested for specificity and amplification efficiency by PCR on the basis of a qRT-PCR standard amplification and dissociation curve. All Ct values were normalized against 18S, and the mean expression values were calculated by using one single gene transcript level from the same group as reference. Sequences of primers are as follows: 18Sfw: CGGCTACCACATCCAAGGAA, rv: GCTGGAATTACCGCGGCT; Matn2fw: GAGGGCTTTGTCCTAGCTGA, rv: GGGAAACTGCCAAGGAATCT; VWFfw: GTCCCAAGAGGAAGTGGACA, rv:
CTCGTGGTACACGACAGAGC; Spink8fw: GACCGGGGAGCTTCTACAAA, rv: AGAATTCCGGAGCAGAGATG; Pkp2fw: TCAGCATACACGGAAGATGC, rv: GCAGAgGCTGTGgTtTCAAT; ApoDfw: AAGATCCCAGCGAGCTTTG, rv: TGAGACGTTGCTCTGTTTGG; Fkbp5fw: AGGTGTTGGCAGTCAATCC, rv: CTTTGGCCTTTCCTTCTTCC; Fnfw: TGACAACTGCCGTAGACCTG, rv: TCGTCTCTGTCAGCTTGCAC; 33 -Tubulinfw: AAGGCCTTCCTGCACTGG, rv: TCTCGGCCTCGGTGAACTC; Il-1ßfw: AGTTGACGGACCCCAAAAGA, rv: GGACAGCCCAGGTCAAAGG; iNOSfw: GGATCTTCCCAGGCAACCA, rv: AATCCACAACTCGCTCCAAGATT; CD11bfw: AAGTGGAGCCATATGAAGTTCACA, rv: GCACCAGGCCCCCAAT; CD45fw: GCGGTGTAAAACTCGTCAAAAAT, rv: GCTTAGGCGTTTCTGGAATCC; TNF-afw: CACAAGATGCTGGGACAGTGA，rv: TCCTTGATGGTGGTGCATGA; Il6fw: CAAAGCCAGAGTCCTTCAGAG, rv: TGGTCCTTAGCCACTCCTTC; Cox2fw: CTCCCTGAAGCCGTACACAT, rv: GCTCGGCTTCCAGTATTGAG; Matn2WT/KO fw: TGTTTGGGACCTGAAAAATCA, Matn2WT rv: CAGTGCAGTCTGCGGATACAT; Matn2KO rv: CCTTTGCAGGTGTATCTTATA, Nlrp3fw: ATTACCCGCCCGAGAAAGG, Nlrp3rv: TCGCAGCAAAGATCCACACAG.

Western blotting. Protein lysates from tissue were prepared using the TissueLyzer II (Qiagen) and RIPA lysis buffer (1\% v/v Triton $\mathrm{X}-100,1 \% \mathrm{w} / \mathrm{v}$ sodium deoxycholate, $0.1 \% \mathrm{w} / \mathrm{v}$ SDS, $50 \mathrm{mM} \mathrm{NaCl}$, $10 \mathrm{mM}$ Tris [pH 7.5]) supplemented with protease and phosphatase inhibitor tablets (Roche). Lysates from primary cells were washed, and lysates were collected with $100 \mu \mathrm{l}$ RIPA lysis buffer using cell scrapers. Lysates were separated under reducing or nonreducing conditions by sodium dodecyl sulphate polyacrylamide gel electrophoresis $(10 \%$ gels) and transferred to PVDF membranes (Pall Corporation) at $25 \mathrm{~V}$ for 2 hours or to nitrocellulose membranes by iBlot (Invitrogen). Proteins were visualized and quantified using the Odyssey Infrared Imaging System and quantification tools (LI-COR Biosciences) or visualized using Amersham Hyperfilm ECL chemiluminescent substrate (Amersham Biosciences). Primary antibodies were MATN2 (R\&D Systems, rabbit), $\beta$-actin (Sigma-Aldrich), pERK1/2 (Cell signaling, rabbit), ERK1/2 (Cell signaling, rabbit), pNF- $\kappa B$ (Cell signaling, rabbit), and NF- $\kappa B$ (Santa Cruz Biotechnology, rabbit).

ELISA for pNfH. Blood samples were collected at the time of culling and were used in an ELISA for pNfH as previously described (6). Antibodies used included chicken anti-pNfH capture and rabbit antipNfH detection. The pNfH ELISA reagents were a gift from Gerry Shaw (Encor Biotechnology, Gainesville, Florida, USA).

Immunofluorescence. Mice were perfused with $20 \mathrm{ml}$ MT-PBS followed by $20 \mathrm{ml} 4 \%$ PFA and postfixed in 4\% PFA for another $30 \mathrm{~min}$ utes (spinal cords) or 1 hour (brains) at $4^{\circ} \mathrm{C}$ and cryosectioned (10-12 $\mu \mathrm{m})$. Frozen sections were rehydrated, blocked with $10 \%$ (v/v) normal serum/0.5\% (v/v) Triton X-100 in MT-PBS for 1 hour, and incubated with primary antibodies at $4^{\circ} \mathrm{C}$ overnight. Fluorescently labeled secondary antibodies were then added for 30 to 60 minutes at room temperature, followed by labeling with Hoechst33342 (Invitrogen). Images were acquired using a Zeiss Axioplan2 upright microscope and Axiovision software. Primary antibodies used were CC1 (Calbiochem, mouse), CD3 (Dako, rabbit), Fn (Chemicon, rabbit), GFAP (Dako, rabbit), IBA-1 (Wako Pure Chemical Industries, rabbit), Lam (Chemicon, rabbit), MATN2 (R\&D Systems, goat), MATN2 (generated in-house; ref. 18; rabbit), NeuN (Chemicon, mouse), SMI312 (Abcam, mouse). In all presented images, MATN2 (R\&D Systems, goat) was used. 
The percentage of the MATN2-positive area in lesions was determined using MetaMorph software (version 7.7.5, Molecular Devices). Lesions, defined as areas with dense nuclei, were identified by segmenting with the Count Nuclei module and then filtered so that nuclei that were clustered $(<5 \mu \mathrm{m})$ were identified as a lesion. The MATN2-positive area was determined by segmentation of the positive staining area and refining it with the Integrated Morphometric Analysis function.

Methylene blue myelin staining. Four spinal cords per genotype were collected and processed as previously described (42). For axonal counts, left and right sections of the dorsal columns of methylene blue-stained spinal cords were photographed at $\times 100$ magnification on a Zeiss Axioplan 2 imaging system. Quantification of axon numbers was performed as previously described (44).

Human tissue and IHC. IHC analyses for MATN2 were performed on brain sections from controls and patients with MS for the investigation of acute demyelinating lesions from biopsy and autopsy tissues; chronic active, chronic inactive, and partly remyelinating lesions from autopsy tissues; and tissues from healthy brain autopsy samples. Lesions were classified according to the previously described classification system (45). A total of 13 different lesions from 6 autopsies and 5 biopsy samples from patients with MS, with lesions located in different parts of the brain, were analyzed. Five tissue samples from gray and white matter areas were selected from brain sections of 3 cases, with no obvious brain pathology to be used as controls.

Human biopsy specimens were fixed in $4 \%$ paraformaldehyde and embedded in paraffin. Autopsy material was fixed in $10 \%$ formalin and tissue embedded in paraffin. Both tissues were cut in $4-\mu$ m-thick sections. Antigen retrieval was performed using either Proteinase $\mathrm{K}$ (Dako) for 10 minutes (MATN2) or citric acid (Thermo Fisher Scientific) for 35 minutes (GFAP, rabbit). After incubation in $3 \% \mathrm{H}_{2} \mathrm{O}_{2}$ and primary antibody at $4^{\circ} \mathrm{C}$ overnight, proteins were visualized using the Vectastain Elite ABC Universal Kit (Vector Laboratories) according to the manufacturer's instructions. Slides were counterstained with Hematoxylin. Primary antibodies were against human MATN2 (R\&D Systems) and GFAP (Dako, rabbit). Controls omitting the primary antibody showed no staining.

Analysis of EAE disease severity in Matn2 KO mice. Absolute lesion size, average nuclei per lesion, and lesion area relative to total tissue area were determined on Hoechst33342-stained (Molecular Probes) sections using MetaMorph. Nuclei counts were based on a standard area count method.

For the quantification of macrophages and $\mathrm{T}$ cells, sections were labeled with Hoechst33342 and IBA-1 or CD3 and analyzed with MetaMorph. IBA-1- and CD3-positive cells were detected using a standard intensity threshold that overlapped or surrounded nuclei (Integrated Morphometric Analysis function). All counts and analyses were performed blinded to the genotype of the animal.

For the described procedures, a minimum of 3 slides containing 20 transverse cords of mixed genotypes were analyzed. Spinal cords were cryosectioned (at $12 \mu \mathrm{m}$ ) $50 \mu \mathrm{m}$ apart to cover a minimum crosssectional area of $200 \mu \mathrm{m}$ of the lumber expansion. Images were automatically captured with a $\times 20$ PlanFluor 0.5 NA objective using the automated Nikon Ti-E microscope.

Primary neuronal cultures. Primary neuronal cultures were derived from embryonic day 16 C57BL/6 embryos. Therefore, cerebral cortices were dissected and the meninges were removed. Cells were dis- sociated by trypsinization (0.125\% Trypsin $/ \mathrm{Ca}^{++}$-free $\mathrm{Mg}^{++}$-free HBSS buffer [Gibco]) for 12 minutes at $37^{\circ} \mathrm{C} .10 \mathrm{ml}$ DMEM/10\% FBS was added, and the tissue was centrifuged at $1,300 \mathrm{~g}$ for 2 minutes. Cells were triturated following addition of neurobasal medium (Invitrogen) containing 1:50 B27 (Invitrogen), 1\% penicillin/streptomycin (Gibco), and $0.25 \%$ GlutaMax (Gibco) and centrifuged at 1,300 $g$ for 2 minutes. Cells were then resuspended in $2 \mathrm{ml} \mathrm{NBM}$ media for plating. The neurons were plated onto poly-D-lysine-coated (Sigma-Aldrich) 6-well plates at a density of $5 \times 10^{5}$ neurons per well. Cultures were maintained in $\mathrm{NBM}$ at $37^{\circ} \mathrm{C} / 5 \% \mathrm{CO}_{2}$. Every 3 to 4 days, $50 \%$ of the media was replenished with fresh medium. Before the performance of assays, floating dead cells in the cultures were removed.

Bone marrow-derived macrophage cultures. Macrophages cultures were prepared as previously described (46). Briefly, femur and tibia were excised, and bone marrow was flushed out. Cells were collected by centrifugation at $350 \mathrm{~g}$ for 5 minutes and then resuspended in macrophage medium (DMEM [Invitrogen no. 11995]; 10\% FCS, $100 \mathrm{U} / \mathrm{ml}$ penicillin, $100 \mu \mathrm{g} / \mathrm{ml}$ streptomycin, and $2 \mathrm{mM}$ GlutaMax-1 [all Gibco]; and 2.5 U human MCSF-1 [R\&D Systems]) and seeded onto a noncoated T175 tissue culture flask for 3 to 4 days. After 3 days, nonadherent, undifferentiated cells were collected and centrifuged at $350 \mathrm{~g}$ for 7 minutes. Cells were cultured for an additional 3 to 4 days in macrophage medium before they were used in experiments. Cells were maintained at $37^{\circ} \mathrm{C}$ at $5 \% \mathrm{CO}_{2}$, and full media change was performed every third day. For the activation of macrophages, recombinant mouse MATN2 (R\&D Systems) was added at concentrations of either 1 to $2 \mu \mathrm{g} / \mathrm{ml}$ for RNA assays or $2 \mu \mathrm{g} / \mathrm{ml}$ for protein assays to the culture media and incubated at $37^{\circ} \mathrm{C} / 5 \% \mathrm{CO}_{2}$ for 4 to 24 hours (RNA) or 15 to 45 minutes (protein).

Primary neuron/microglia cocultures. After 7 to 8 days in culture, neurons were cocultured with macrophages by placing the macrophages onto the neurons. Therefore, macrophages were collected by centrifugation at $350 \mathrm{~g}$ for 7 minutes, resuspended in $1 \mathrm{ml}$ neuronal medium, counted, and added to the neurons at 350,000 cells per well in the 6-well plates.

For the activation of macrophages, $1 \mu \mathrm{g} / \mathrm{ml}$ LPS (Sigma-Aldrich) was added to the culture media and cocultures were incubated at $37^{\circ} \mathrm{C} / 5 \% \mathrm{CO}_{2}$ for 24 hours. Where indicated, instead of the LPS recombinant MATN2 (R\&D Systems) at $1 \mu \mathrm{g} / \mathrm{ml}$ was added to the cultures.

Live/dead assay and axonal injury analysis. Cells were incubated with Calcein AM and Ethidium Homodimer ( $4 \mu \mathrm{M}$; Invitrogen) for 12 minutes at $37^{\circ} \mathrm{C}$ followed by Hoechst33342 for an additional 5 minutes. Images of 5 fields of view were randomly chosen and captured from each well. To determine cell viability, cells were counted using the cell count function in MetaMorph software. For identification of neurite length and branching, the neurite outgrowth module within MetaMorph was used. This module identifies neurons using the Calcein AM stain via their nuclei and traces neurites to determine the neurite outgrowth per cell and number of branches per cell. The following parameters were used to identify the cell bodies and neurites: cell bodies $=30 \mu \mathrm{m}$, maximum width of neurites $=6 \mu \mathrm{m}$.

TLR activation assay. MATN2-induced stimulation was tested in HEK293 cells expressing one of the TLRs (TLR2, TLR3, TLR4, TLR5, TLR7, TLR8, or TLR9) and secreted alkaline phosphatase under the control of a modified human $I l 12 p 40$ promoter, which is inducible by $\mathrm{NF}-\kappa \mathrm{B}$, activator protein-1, and IFN-regulatory factors cloned into pNIFTY2-SEAP vectors. The amount of secreted alkaline phosphatase 
was determined using the SEAP Reporter Assay Kit (measurements were performed by TLR-screening service of InvivoGen) as arbitrary units. Screening was performed in triplicates using $2 \mu \mathrm{g} / \mathrm{ml}$ MATN2 and was compared with the induction of alkaline phosphatase in noninduced TLR clones and NF- $\kappa \mathrm{B}$-negative cells using TNF- $\alpha$ (negative controls) as well as to the activation with stimulating ligands (positive controls): TLR2 = heat-killed Listeria monocytogenes at $10^{8}$ cells per $\mathrm{ml}$; $\mathrm{TLR} 3=\operatorname{poly}(\mathrm{I}: \mathrm{C})$ at $1 \mu \mathrm{g} / \mathrm{ml}$; TLR4 $=$ E. coli $\mathrm{K} 12 \mathrm{LPS}$ at $100 \mathrm{ng} / \mathrm{ml}$; TLR5 $=$ S. typhimurium flagellin at $100 \mathrm{ng} / \mathrm{ml}$; TLR7 = CL097 at $1 \mu \mathrm{g} / \mathrm{ml}$; TLR8 = CL075 at $10 \mu \mathrm{g} / \mathrm{ml}$ plus poly $(\mathrm{dT}) 10 \mu \mathrm{M}$; and TLR9 = CpG ODN 1826 at $100 \mathrm{ng} / \mathrm{ml}$ (InvivoGen).

Statistics. Statistical analysis was conducted using GraphPad prism software V.6 (GraphPad Software). Values are expressed as mean \pm SEM. Groups of 2 were compared by unpaired 2-tailed $t$ test. One- or two-way ANOVA followed by Bonferroni's multiple comparison test were used for comparisons among three or more groups. For EAE disease curves, Mann-Whitney rank-sum tests were performed. In Kaplan-Meier curve, a $\chi^{2}$ test was used for statistical analysis. A $P$ value of less than 0.05 was considered statistically significant.

Study approval. All animal experimentation was conducted according to National Health and Medical Research Council guidelines (Australia) and with approval from the Howard Florey Institute animal ethics committee (09-113).

Human histological studies were conducted in the laboratory of T. Kuhlmann. All patient biopsies were undertaken for clinical indications after standard clinical consent was obtained and documented. Autopsies were conducted with consent of relatives. These materials were stored in standard pathology archives. The ethics committee of the University Hospital, Muenster, has approved the use of these materials for scientific purposes. According to German law, patients and relatives do not have to explicitly consent to the use of these codified materials for specific scientific studies, on the proviso that all publication of data respects patient anonymity.

\section{Acknowledgments}

The authors would like to thank Elke Hoffmann and Claudia Kemming (Institute for Neuropathology, Muenster, Germany) for assisting with the human IHC processing. This work was supported by the National Multiple Sclerosis Society (US) (project grant RG3850A3/1 to H. Butzkueven and T. Kilpatrick); National Health and Medical Research Council Australia (project grant 509088 to H. Butzkueven and T. Kilpatrick, Career Development Award to H. Butzkueven, Centres of Research Excellence grant 1001216 providing fellowship support to M. Gresle); and a private donation form Kate and Gavin Rogers. R. Wagener was supported by the Deutsche Forschungsgemeinschaft grant WA 1338/2-6. B. Emery is supported by an National Health and Medical Research Council Australia career development fellowship. The funders had no role in the study design, data collection and analysis, decision to publish, or preparation of the manuscript.

Address correspondence to: Helmut Butzkueven, Melbourne Brain Centre, 4 Centre, Royal Melbourne Hospital, Parkville, Victoria, Australia, 3050. Phone: 61.393424402; E-mail: butz@unimelb.edu.au.
1. Trapp BD, Peterson J, Ransohoff RM, Rudick R, Mörk S, Bö L. Axonal transection in the lesions of multiple sclerosis. N Engl J Med. 1998;338(5):278-285.

2. Kornek B, et al. Multiple sclerosis and chronic autoimmune encephalomyelitis: a comparative quantitative study of axonal injury in active, inactive, and remyelinated lesions. Am J Pathol. 2000;157(1):267-276.

3. Lovas G, Szilágyi N, Majtényi K, Palkovits M, Komoly S. Axonal changes in chronic demyelinated cervical spinal cord plaques. Brain. 2000;123(pt 2):308-317.

4. Lucchinetti CF, et al. Inflammatory cortical demyelination in early multiple sclerosis. $N$ Engl J Med. 2011;365(23):2188-2197.

5. Dutta R, et al. Activation of the ciliary neurotrophic factor (CNTF) signaling pathway in cortical neurons of multiple sclerosis patients. Brain. 2007;130(pt 10):2566-2576.

6. Gresle MM, et al. Validation of a novel biomarker for acute axonal injury in experimental autoimmune encephalomyelitis. J Neurosci Res. 2008;86(16):3548-3555.

7. Butzkueven H, Emery B, Cipriani T, Marriott MP, Kilpatrick TJ. Endogenous leukemia inhibitory factor production limits autoimmune demyelination and oligodendrocyte loss. Glia. 2006;53(7):696-703.

8. Palace J. Neuroprotection and repair. J Neurol Sci. 2008;265(1-2):21-25.

9. Schaefer L, et al. The matrix component biglycan is proinflammatory and signals through Toll-like receptors 4 and 2 in macrophages. JClin Invest.
2005;115(8):2223-2233.

10. Merline R, et al. Signaling by the matrix proteoglycan decorin controls inflammation and cancer through PDCD4 and microRNA-21. Sci Signal. 2011;15(199):ra75

11. Kim S, et al. Carcinoma-produced factors activate myeloid cells through TLR2 to stimulate metastasis. Nature. 2009;457(7225):102-106.

12. Termeer C, et al. Oligosaccharides of Hyaluronan activate dendritic cells via toll-like receptor 4 . J Exp Med. 2002;195(1):99-111.

13. Moreth $\mathrm{K}$, et al. The proteoglycan biglycan regulates expression of the $\mathrm{B}$ cell chemoattractant CXCL13 and aggravates murine lupus nephritis. J Clin Invest. 2010;120(12):4251-4272.

14. Schaefer L, Iozzo RV. Small leucine-rich proteoglycans, at the crossroad of cancer growth and inflammation. Curr Opin Genet Dev. 2012;22(1):56-57.

15. Schaefer L. Small leucine-rich proteoglycans in kidney disease. J Am Soc Nephrol. 2011;22(7):1200-1207.

16. Schaefer L. Extracellular matrix molecules: endogenous danger signals as new drug targets in kidney diseases. Curr Opin Pharmacol. 2010;10(2):185-190.

17. Klatt AR, Becker AK, Neacsu CD, Paulsson M, Wagener R. The matrilins: modulators of extracellular matrix assembly. Int J Biochem Cell Biol. 2011;43(3):320-330.

18. Piecha D, et al. Matrilin-2, a large, oligomeric matrix protein, is expressed by a great variety of cells and forms fibrillar networks. J Biol Chem. 1999;274(19):13353-13361.

19. Segat D, et al. Expression of matrilin-1, -2 and
-3 in developing mouse limbs and heart. Matrix Biol. 2000;19(7):649-655.

20. Piecha D, et al. Expression of matrilin-2 in human skin. J Invest Dermatol. 2002;119(1):38-43.

21. Szabo E, et al. Expression of matrilin-2 in liver cirrhosis and hepatocellular carcinoma. Pathol Oncol Res. 2008;14(1):15-22.

22. Malin D, et al. The extracellular-matrix protein matrilin-2 participates in peripheral nerve regeneration. J Cell Sci. 2009;122(pt 7):995-1004.

23. Comabella M, Martin R. Genomics in multiple sclerosis-current state and future directions. J Neuroimmunol. 2007;187(1-2):1-8.

24. Linker RA, Lee DH. Models of autoimmune demyelination in the central nervous system: on the way to translational medicine. Exp Transl Stroke Med. 2009;1:5.

25. Mátés L1, Nicolae C, Mörgelin M, Deák F, Kiss I, Aszódi A. Mice lacking the extracellular matrix adaptor protein matrilin-2 develop without obvious abnormalities. Matrix Biol. 2004;23(3):195-204.

26. Muratoglu S, et al. Primary structure of human matrilin-2, chromosome location of the MATN2 gene and conservation of an AT-AC intron in matrilin genes. Cytogenet Cell Genet. 2000;90(3):323-327.

27. Deák F, Piecha D, Bachrati C, Paulsson M, Kiss I. Primary structure and expression of matrilin-2, the closest relative of cartilage matrix protein within the von Willebrand factor type A-like module superfamily. J Biol Chem. 1997;272(14):9268-9274.

28. Strand AD, et al. Conservation of regional gene expression in mouse and human brain. PLoS 
Genet. 2007;3(4):e59.

29. Baeten KM, Akassoglou K. Extracellular matrix and matrix receptors in blood-brainbarrier formation and stroke. Dev Neurobiol. 2011;71(11):1018-1039.

30. Babelova A, et al. Biglycan, a danger signal that activates the NLRP3 inflammasome via toll-like and P2X receptors. J Biol Chem. 2009;284(36):24035-24048.

31. Magalhães PO, et al. Methods of endotoxin removal from biological preparations: a review. JPharm Pharm Sci. 2007;10(3):288-404.

32. Popovic ZV, et al. The proteoglycan biglycan enhances antigen-specific $T$ cell activation potentially via MyD88 and TRIF pathways and triggers autoimmune perimyocarditis. JImmunol. 2011;187(12):6217-6226.

33. Chen GY, Nuñez G. Sterile inflammation: sensing and reacting to damage. Nat Rev Immunol. 2010;10(12):826-837.

34. Dinarello CA. A clinical perspective of IL-1 $\beta$ as the gatekeeper of inflammation. Eur J Immunol. 2011;41(5):1203-1217.

35. Akira S, Takeda K. Toll-like receptor signaling.
Nat Rev Immunol. 2004;4(7):499-511.

36. Derbali $\mathrm{H}$, et al. Increased biglycan in aortic valve stenosis leads to the overexpression of phospholipid transfer protein via Toll-like receptor 2. Am J Pathol. 2010;176(6):2638-2645.

37. Moreth K, Iozzo RV, Schaefer L. Small leucine-rich proteoglycans orchestrate receptor crosstalk during inflammation. Cell Cycle. 2012;11(11):2084-2091.

38. Tang SC, et al. Pivotal role for neuronal Tolllike receptors in ischemic brain injury and functional deficits. Proc Natl Acad Sci U S A. 2007;104(34):13798-13803.

39. Mattson MP, Maudsley S, Martin B. A neural signaling triumvirate that influences ageing and age-related disease: insulin/IGF-1, BDNF and serotonin. Ageing Res Rev. 2004;3(4):445-464.

40. Mattson MP, Barger SW, Begley JG, Mark RJ. Calcium, free radicals and ecitotoxic neuronal death in primary cell culture. Methods Cell Biol. 1995;46:187-216.

41. Adachi O, et al. Targeted disruption of the MyD88 gene results in loss of IL-1- and IL-18-mediated function. Immunity. 1998;9(1):143-150.
42. Gresle MM, et al. Leukemia inhibitory factor protects axons in experimental autoimmune encephalomyelitis via an oligodendrocyte-independent mechanism. PLoS One. 2012;7(10):e47379.

43. Livak KJ, Schmittgen TD. Analysis of relative gene expression data using real-time quantitative PCR and the 2(-Delta Delta C(T)) Method. Methods. 2001;25(4):402-408.

44. Munro KM, et al. EphA4 receptor tyrosine kinase is a modulator of onset and disease severity of experimental autoimmune encephalomyelitis (EAE). PLoS One. 2013;8(2):e55948.

45. Lassmann H. Review: the architecture of inflammatory demyelinating lesions: implications for studies on pathogenesis. Neuropathol Appl Neurobiol. 2011;37(7):698-710.

46. Fleetwood AJ, Lawrence T, Hamilton JA, Cook AD. Granulocyte-macrophage colony-stimulating factor (CSF) and macrophage CSF-dependent macrophage phenotypes display differences in cytokine profiles and transcription factor activities: implications for CSF blockade in inflammation. JImmunol. 2007;178(8):5245-5252. 